\title{
Formation of Block Copolymer Micelles in Solution: A Monte Carlo Study of Chain Length Dependence
}

\author{
Andrey Milchev, ${ }^{\dagger, \ddagger}$ Aniket Bhattacharya, ${ }^{\mathrm{t}, \S}$ and Kurt Binder $*, t$ \\ Institut für Physik, J ohannes Gutenberg-Universität, Mainz, Staudinger Weg 7, \\ D-55099 Mainz, Germany; Institute for Physical Chemistry, Bulgarian Academy of Sciences, \\ 1113, Sofia, Bulgaria; and Department of Physics, Michigan State University, \\ East Lansing, Michigan 48824-1116
}

Received April 13, 2000; Revised Manuscript Received October 25, 2000

\begin{abstract}
Short block copolymers in a selective solvent (bad for A-block, good for B-block) are modeled by flexible bead-spring chains, where beads interact with short-range Morse potentials of variable strength. In particular, treating the strength $\mathrm{E}_{\mathrm{AA}}$ of attraction between monomers of the A-block as a variable, we study the mass distribution of the micel les that are formed under conditions that correspond to the vicinity of the critical micelle concentration (cmc). Choosing a composition $f=N_{A} / N=1 / 4$ for the block copolymers, we vary their chain length $\mathrm{N}$ from $\mathrm{N}=4$ to $\mathrm{N}=32$. Only such relatively short chains can be used in thermal equilibrium, since the relaxation times of the system increase dramatically with increasing length. We show that in the regime of parameters accessible to our study, the number of chains per mi celle is rather small and almost independent of chain length, implying that the core radius scales as $\mathrm{N}_{\mathrm{A}}^{1 / 3}$ in this regime. We compare our results with existing theoretical predictions and with experiments.
\end{abstract}

\section{Introduction}

The formation of micelles from amphiphilic molecules in solution is important for many applications in biological and industrial processes. ${ }^{1-7}$ At the same time, the theory of micelle formation within the framework of statistical thermodynamics is a challenging and longstanding problem which still is not fully understood. ${ }^{8-30}$ Particularly interesting in this context are block copolymers ${ }^{9,11,14,18,19,23-29} A_{f} B_{1-f}$ in solution, because both the composition $f=N_{A} / N$ and the chain length $\mathrm{N}\left(=\mathrm{N}_{\mathrm{A}}+\mathrm{N}_{\mathrm{B}}\right)$ of the total macromolecule can be varied over a wide range, ${ }^{28,31-36}$ without affecting intermolecular forces significantly. Thus, choosing $\mathrm{f}$ and $\mathrm{N}$ as control parameters in experiments, one would perform a stringent test of the theories which make interesting predictions how the linear dimensions of the micelles and the number of chains per micelle depend on these parameters. ${ }^{9,11,14,18,23-27}$ In fact, this point has al ready been made by several authors, and systemspecific predictions ${ }^{27}$ have been made for several blockcopolymer-solvent systems and also carried out a comparison with experiment. ${ }^{37}$ Further experimental studies where these theoretical predictions are tested can be found in refs 38-45. Such studies are also clearly difficult to perform, since the critical micelle concentration $\left(\phi_{\mathrm{cmc}}\right)$ seems to decrease al most exponentially with $\mathrm{N}$, at least according to the model calculations. ${ }^{23-27}$

Thus, it would be very interesting to complement the experiments by computer simulations of model systems, since in computer simulations it is possible al so to vary intermolecular forces at will in a well-controlled manner ${ }^{46-48}$ In fact, a large number of computer simulations of micelle formation can al ready be found in the literature. ${ }^{49-67}$ However, only recently has it been

* To whom correspondence should be addressed: E-mail: kurt.binder@uni-mainz.de

† J ohannes Gutenberg-Universität.

‡ Bulgarian Academy of Sciences.

$\S$ Michigan State University. clearly realized that for a study of a well-equilibrated micellar size distribution in solution one needs huge systems with a large number of micelles in them simulated over a sufficient time range so that the molecules get exchanged between the micelles often enough. ${ }^{63,65}$ These studies 63,65 hence applied coarsegrained models of block copolymers as short as $\mathrm{N}=4$ only! The question of how the surfactant chain length affects the behavior was already discussed by Palmer and Liu, 62 but some aspects of their results were rather inconclusive: As noted by these authors, the long lifetime of the clusters, combined with the small number of clusters present in each of the simulations, indicated that the calculated distributions did not reflect the equilibrium distribution that would be seen for an infinite system. ${ }^{62}$ While these authors worked for $\mathrm{N}=$ 4, 6, 8 with only 100 surfactant molecules in their simulation box, we hence use, for $N=4, n=1024$ block copolymers in our system and $\mathrm{n}=512$ for $\mathrm{N}=8$, varying the block copolymer chain length $\mathrm{N}$ from $\mathrm{N}=4$ to $\mathrm{N}=$ 32 at fixed monomer density $\phi=0.125$ (in our units, this corresponds to about $1 / 16$ th of the density of a dense melt). ${ }^{68}$ Nevertheless, the small number of micelles in the simulation box is clearly also a problem for the simulations that will be presented below. An even more serious problem may be the shortness of our chains, and it seems doubtful to what extent there is a relation to theories that exploit the statistical properties of very long chains. However, the coarse-grained model which is used here implies that every bond al ready represents an effective segment (Kuhn segment) of a flexible chain; therefore properties such as the scal ing of the gyration radius in dense melts $\left(R_{g} \propto \sqrt{N}\right)$ hold for extremely small $N(N<10)$ al ready; al so the single-chain structure factor of a homopolymer is almost indistinguishable from the Debye function for $\mathrm{N}=10$ al ready. ${ }^{46} \mathrm{Although}$ this is not a guarantee that for the present problem our chains are long enough to allow a meaningful test of the theories, it is neverthel ess interesting to study also micelles formed from short block copolymers, since these 
systems are of interest experimentally.

Our paper is organized as follows: in section 2, we recall the main theoretical predictions, while section 3 describes the main features of the model that is simulated. The results of our work are discussed in section 3.1, and some conclusions finally are summarized in section 4 where also a comparison of our findings with various experiments is given.

\section{Brief Review of the Phenomenological Theories of the Formation of Block Copolymer Micelles}

Following standard practice, $18,22,27$ we treat the micellar solution as a multicomponent solution, where $\mathrm{N}_{\mathrm{s}}$ solvent molecules, $\mathrm{N}_{1}$ single blockcopolymers ("unimers") and $N_{n}$ micelles of aggregation number $n(2 \leq n<\infty)$ are present. Each of the above species is treated as a distinct chemical component, and the Gibbs free energy $\mathrm{G}$ of the system is

$$
\mathrm{G}=\mathrm{G}_{\mathrm{ref}}+\mathrm{G}_{\mathrm{ent}}+\mathrm{G}_{\mathrm{int}}
$$

The first term represents the standard state corresponding to infinitely dilute solution conditions, $G_{\text {ent }}$ accounts for the entropy of the multicomponent system, and $G_{i n t}$ accounts for the mutual interactions among all the species. Invoking random mixing, this term is

$$
\mathrm{G}_{\text {int }}=\mathrm{k}_{\mathrm{B}} \mathrm{T} \chi_{\mathrm{s}} \mathrm{N}_{\mathrm{s}} \phi
$$

where $\chi_{\mathrm{s}}$ is a Flory-Huggins type interaction parameter, and $\phi$ is the volume fraction of the block copolymers

$$
\phi=\sum_{n=1}^{\infty} \frac{\mathrm{nN}_{\mathrm{n}}}{\left(\mathrm{N}_{\mathrm{s}}+\sum_{\mathrm{n}=1}^{\infty} \mathrm{nN}_{\mathrm{n}}\right)}
$$

where the molecular volume of a solvent molecule is assumed equal to that of a monomer of the block copolymer.

For the estimation of $G_{\text {ent }}$, one may, ${ }^{27}$ simply use the Flory-Huggins model 69

$$
\mathrm{k}_{\mathrm{B}} T\left[\mathrm{~N}_{\mathrm{s}} \ln \left(\frac{\mathrm{N}_{\mathrm{s}}}{N_{\mathrm{s}}+\sum_{n=1}^{\infty} \mathrm{nN} N_{n}}+\sum_{n=1}^{\infty} N_{n} \ln \frac{\mathrm{nN}_{\mathrm{n}}}{N_{\mathrm{s}}+\sum_{n=1}^{\infty} n N_{n}}\right)\right]
$$

Finally, the reference state Gibbs potential is written as

$$
\mathrm{G}_{\mathrm{ref}}=\mathrm{N}_{\mathrm{s}} \mu_{\mathrm{s}}^{\circ}+\sum_{\mathrm{n}=1}^{\infty} \mathrm{N}_{\mathrm{n}} \mu_{\mathrm{n}}^{\circ}
$$

where $\mu_{\mathrm{s}}^{\circ}$ is the chemical potential of pure solvent, $\mu_{1}^{\circ}$ and $\mu_{\mathrm{n}}^{\circ}$ are the chemical potentials of a single block copolymer and an aggregate containing $n$ block copolymers at infinite dilution. From the Gibbs-Duhem relation valid for any multicomponent system

$$
\mathrm{G}=\mathrm{N}_{\mathrm{s}} u_{\mathrm{s}}+\sum_{\mathrm{n}=1}^{\infty} \mathrm{N}_{\mathrm{n}} \mu_{\mathrm{s}}
$$

one finds from eqs 2.1 to 2.5 that

$$
\begin{aligned}
\mu_{\mathrm{n}}=\mu_{\mathrm{n}}^{\circ}+\mathrm{k}_{\mathrm{B}} T\left(\ln \left(\frac{\mathrm{nN} \mathrm{N}_{\mathrm{n}}}{N_{\mathrm{s}}+\sum_{\mathrm{n}^{\prime}} \mathrm{n}^{\prime} \mathrm{N}_{\mathrm{n}^{\prime}}}\right)+\right. \\
1-\mathrm{n}\left(\frac{\left.\mathrm{N}_{\mathrm{s}}+\sum_{\mathrm{n}^{\prime}} \mathrm{N}_{\mathrm{n}^{\prime}}\right)}{\left.\mathrm{N}_{\mathrm{s}}+\sum_{\mathrm{n}^{\prime}} \mathrm{n}^{\prime} \mathrm{N}_{\mathrm{n}^{\prime}}\right)}+\mathrm{nk}_{\mathrm{B}} T \chi_{\mathrm{s}}\left(\frac{\mathrm{N}}{\mathrm{N}_{\mathrm{s}}+\sum_{\mathrm{n}^{\prime}} \mathrm{n}^{\prime} \mathrm{N}_{\mathrm{n}^{\prime}}}\right)^{2},\right.
\end{aligned}
$$

Now in equilibrium, $\delta \mathrm{G}=0$, obeying the constraints $\delta \mathrm{N}_{\mathrm{s}}$ $=0$ and $\delta\left(\sum_{n=1}^{\infty} \mathrm{nN}_{\mathrm{n}}\right)=0$. This yields the fundamental condition

$$
\mu_{\mathrm{n}} / \mathrm{n}=\mu_{1}
$$

i.e., the chemical potential of the single dispersed molecule is equal to the chemical potential per molecule of aggregates of any size $n$. Equations 2.7 and 2.8 yield the size distribution of micelles

$$
\phi_{\mathrm{n}}=\phi_{1}{ }^{\mathrm{n}} \exp (\mathrm{n}-1) \exp \left[-\left(\frac{\mu_{\mathrm{n}}^{\circ}-\mathrm{n} \mu_{1}^{\circ}}{\mathrm{k}_{\mathrm{B}} \mathrm{T}}\right)\right]
$$

where $\phi_{n}=n N_{n} /\left(N_{s}+\sum_{n^{\prime}=1}^{\infty} n^{\prime} N_{n^{\prime}}\right)$ is the vol ume fraction of species $\mathrm{n}$.

Near the critical micelle concentration $\phi_{\mathrm{cmc}}, \phi_{\mathrm{n}}$ is sharply peaked at an aggregation number $\bar{n} \gg 1$. Then $\phi_{\tilde{n}} \ll \phi_{1}$ and eq 2.9 can be simplified as follows

$\mu_{1}^{\circ}+\mathrm{k}_{\mathrm{B}} \mathrm{T} \ln \left(\mathrm{e} \phi_{1}\right)=\left[\mu_{\mathrm{n}}^{\circ}+\mathrm{k}_{\mathrm{B}} \mathrm{T} \ln \left(\phi_{\mathrm{n}} \mathrm{e}\right)\right] / \mathrm{n} \approx \mu_{\mathrm{n}}^{\circ} / \mathrm{n}$

This equation describes the micelle as a "pseudophase" in equilibrium with single dispersed copolymer molecules. Since at equilibrium the free energy per molecule of a phase is a minimum, $\bar{n}$ is found from

$$
\frac{\partial}{\partial \mathrm{n}}\left(\mu_{\mathrm{n}}^{\circ} / \mathrm{n}-\mu_{1}^{\circ}\right)=0, \quad \mathrm{n}=\overline{\mathrm{n}}
$$

From eq 2.10 one then finds the critical micelle concentration

$$
\phi_{\mathrm{cmc}}=\left.\phi_{1}\right|_{\mathrm{n}=\mathrm{n}}=\frac{1}{\mathrm{e}} \exp \left(\frac{\mu_{\mathrm{n}}^{\circ} / \overline{\mathrm{n}}-\mu_{1}^{\circ}}{\mathrm{k}_{\mathrm{B}} \mathrm{T}}\right)
$$

So far, we just have recalled the standard treatment of micel lization for any amphiphilic mol ecules; the aspects specific for block copolymers enter in the treatments of the chemical potential in eq 2.12. The quantity $\mu_{\mathrm{n}}^{\circ} / \mathrm{n}-$ $\mu_{1}^{\circ}$ represents the change in the reference state free energy when a single block copolymer molecule in solution is transferred into a micelle of aggregation number $\mathrm{n}$. The various theories $9,11,14,18,19,23-27$ differ in their assumptions about this expression, however, although they all deal with micelles of spherical shape (Figure 1). Here we assume that in the micelles the A-monomers in the core are fully segregated from the B-monomers in the corona, and follow de Gennes ${ }^{14}$ assuming that the A-blocks are stretched with an endto-end distance equal to the core radius $\mathrm{R}$ (Figure 1 ). Similarly, the B-chains are stretched over a distance $\mathrm{S}-\mathrm{R}$, the thickness of the corona region. Taking the 


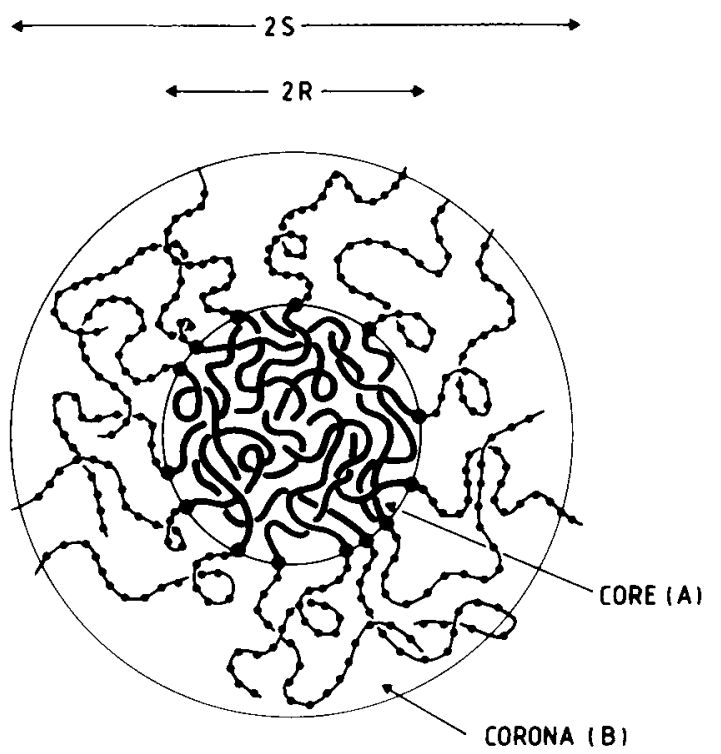

Figure 1. Schematic picture of a spherical block copolymer micelle, assuming strong segregation between the A-blocks (thick lines) that form the micellar core and the B-blocks (chain lines) that form the corona. The A-B junctions (highlighted by large dots) are localized at the surface of the core, which forms a sphere of radius $\mathrm{R}$, while the total micelle forms a sphere of radius $\mathrm{S}$.

density $\rho$ as saturated (i.e., $\rho=1$ ) in the core region, we must have

$$
\mathrm{nN}_{\mathrm{A}}=\frac{4 \pi}{3}(\mathrm{R} / \mathrm{a})^{3}
$$

where a is the size of a monomer. Now Figure 1 implies that there are three contributions to the free energy of a micelle: elastic energies due to the stretching of the A-blocks and the B-blocks, and an interfacial free energy which is $4 \pi \mathrm{R}^{2} \sigma, \sigma$ being the interfacial tension which for large $N_{A}, N_{B}$ in the strong segregation limit is simply related to the Flory-Huggins-parameter $\chi_{A B}$ by which $\mathrm{A}$ and $\mathrm{B}$ monomers repel each other, ${ }^{70} \sigma / \mathrm{k}_{\mathrm{B}} \mathrm{T}=\mathrm{a}^{-2}$ $\sqrt{\chi_{A B} / 6}$. For not too small a value of $f$, one can show ${ }^{14}$ that the relation between $\mathrm{R}$ and $\mathrm{N}_{\mathrm{A}}$ is the same as in strongly segregated lamellar block copolymer phases

$$
\mathrm{R} \propto \mathrm{N}_{\mathrm{A}}^{2 / 3}, \quad \mathrm{n} \propto \mathrm{N}_{\mathrm{A}}, \mathrm{N}_{\mathrm{A}} \rightarrow \infty, \quad \chi_{\mathrm{AB}}{ }^{\text {crit }} \ll \chi_{\mathrm{AB}}
$$

For the weak segregation limit (diffuse $A-B$ interfaces) the A-blocks are not stretched, but rather a Gaussian chain statistics ${ }^{71}$ applies, and hence $e^{9,11,14}$

$$
\mathrm{R} \propto \mathrm{N}_{\mathrm{A}}^{1 / 2}, \quad \mathrm{n} \propto \mathrm{N}_{\mathrm{A}}^{1 / 2}, \quad \mathrm{~N}_{\mathrm{A}} \gg 1, \quad \chi_{\mathrm{AB}} \mathrm{N}_{\mathrm{A}}=\mathrm{o}(1)
$$

Both eqs 2.14 and 2.15 are asymptotic laws which hold in particular limits only, while outside of these limits one may observe "effective exponents" in the relation between $\mathrm{R}$ and $\mathrm{N}_{\mathrm{A}}$ (or between $\mathrm{n}$ and $\mathrm{N}_{\mathrm{A}}$, respectively), and corrections to the leading behavior are expected if $\mathrm{N}_{\mathrm{A}}$ is not large enough.

Leibler et al. ${ }^{18}$ consider only the special case where $f$ $=1 / 2$ and the solvent is made of homopolymers of type $B$ and chain length $\mathrm{N}_{\mathrm{h}}$ : therefore a Flory-Huggins parameter $\chi_{\mathrm{BB}}$ describing the energetic difference of interactions between pairs of monomers of type $B$ and the monomer $\mathrm{B}$-solvent interaction does not arise.
Leibler et al. ${ }^{18}$ decompose $\mu_{\mathrm{n}}^{\circ}$ in three terms

$$
\mu_{\mathrm{n}}^{\circ}=4 \pi \mathrm{R}^{2} \sigma+\mathrm{g}_{\text {def }}+\mathrm{g}_{\text {mix }}
$$

where the first term is the interfacial free energy between micellar core and corona, and the second term is a contribution due to the deformation of copolymer chains, $9,11,14,72$ while the last contribution represents the entropy of mixing of homopolymer chains and B-blocks in the corona

$$
\mathrm{g}_{\text {mix }}=\frac{4 \pi}{3} \frac{\mathrm{S}^{3}-\mathrm{R}^{3}}{\mathrm{a}^{3}} \mathrm{k}_{\mathrm{B}} \mathrm{T} \frac{1-\eta}{\mathrm{N}_{\mathrm{h}}} \ln (1-\eta)
$$

$\eta$ being the volume fraction of B-monomers belonging to copolymers, $1-\eta$ is the rest belonging to the homopolymers. Using an incompressibility condition $\eta$ and $\mathrm{S}$ are related as

$$
4 \pi\left(\mathrm{S}^{3}-\mathrm{R}^{3}\right) \eta / 3=\mathrm{nN}_{\mathrm{B}} \mathrm{a}^{3}
$$

which complements eq 2.13. Finally, the contribution due to deformation is ${ }^{9,11,14,72}$

$$
\begin{aligned}
& g_{\text {def }}= \\
& \quad \frac{3}{2} k_{B} \operatorname{Tn}\left\{\frac{R^{2}}{N_{A} a^{2}}+\frac{N_{A} a^{2}}{R^{2}}+\frac{(S-R)^{2}}{N_{B} a^{2}}+\frac{N_{B} a^{2}}{(S-R)^{2}}-4\right\}
\end{aligned}
$$

Note that this contribution vanishes if the chains take their unperturbed dimensions $\left\{R^{2}=N_{A} a^{2},(S-R)^{2}=\right.$ $\mathrm{N}_{\mathrm{B}} \mathrm{a}^{2}$.

Leibler et al. ${ }^{18}$ then minimize $\mu_{\mathrm{n}}^{\mathrm{o}} / \mathrm{n}$ taking $\mathrm{n}$ and $\eta$ as independent variables. They find from their numerical calculations for relatively small incompatibility between $A$ and $B$ that

$$
\mathrm{R} \propto \mathrm{N}_{\mathrm{A}}^{0.53}, \mathrm{n} \propto \mathrm{N}_{\mathrm{A}}^{0.8}
$$

while for large incompatibility, they confirm eq 2.14.

An alternative treatment of the problem is due to Nagarajan and Ganesh, ${ }^{27}$ who construct $\mu_{\mathrm{n}}^{\circ} / \mathrm{n}-\mu_{1}^{\circ}$ from six individual contributions

$$
\begin{array}{r}
\frac{\mu_{\mathrm{n}}^{\circ}}{\mathrm{n}}-\mu_{1}^{\circ}=4 \pi \mathrm{R}^{2} \sigma+\Delta \mu_{\mathrm{def}, \mathrm{A}}^{\circ}+\Delta \mu_{\mathrm{def}, \mathrm{B}}^{\circ}+\Delta \mu_{\mathrm{dil}, \mathrm{A}}^{\circ}+ \\
\Delta \mu_{\mathrm{dil}, \mathrm{B}}^{\circ}+\Delta \mu_{\mathrm{loc}}^{\circ}
\end{array}
$$

Here $\Delta \mu_{\text {def,A }}^{\circ}$ and $\Delta \mu_{\text {def, } \mathrm{B}}^{\circ}$ account for the change of deformation when a block copolymer is transformed into a micelle (these two terms are the analogue of $g_{\text {def }}$ in eq 2.19), while $\Delta \mu_{\text {dil,A }}^{\circ}$ and $\Delta \mu_{\text {dil, }}^{\circ}$ represent the free energy change due the change of dilution. Finally, $\mu_{\text {loc }}^{\circ}$ accounts for the reduction in configurational surface of the micellar core (Figure 1). In general, the expression resulting from eqs 2.11 and 2.21 must be evaluated numerically. For typical parameters ${ }^{27}$

$$
\begin{aligned}
\mathrm{R} \propto \mathrm{N}_{\mathrm{A}}{ }^{0.70} \mathrm{~N}_{\mathrm{B}}{ }^{-0.08,}, \mathrm{n} \propto \mathrm{N}_{\mathrm{A}}{ }^{1.10} \mathrm{~N}_{\mathrm{B}}{ }^{-0.24}, \\
\mathrm{D} \equiv \mathrm{S}-\mathrm{R} \propto \mathrm{N}_{\mathrm{A}}{ }^{0.07} \mathrm{~N}_{\mathrm{B}}{ }^{0.68}
\end{aligned}
$$

The exponents in eqs 2.20 and 2.22 are "effective exponents" and do not describe the true asymptotic behavior as $\mathrm{N}_{\mathrm{A}} \rightarrow \infty$ at constant f. In particular, the exponents in eq 2.22 do depend on the interaction parameters between monomers $A, B$ and the solvent 
molecules. Nagarajan and Ganesh ${ }^{27}$ observe that their numerical results are well approximated by the following closed-form expression:

$$
\begin{aligned}
& \mathrm{R} / \mathrm{a}= \\
& {\left[3 \mathrm{~N}_{\mathrm{A}}{ }^{2}\left(\sigma_{\mathrm{AS}} \mathrm{a}^{2} / \mathrm{k}_{\mathrm{B}} \mathrm{T}\right)+\mathrm{N}_{\mathrm{A}}{ }^{3 / 2}+\mathrm{N}_{\mathrm{A}} \mathrm{N}_{\mathrm{B}}{ }^{1 / 2}(\mathrm{R} / \mathrm{D})\right]^{1 / 3}} \\
& {\left[1+\mathrm{N}_{\mathrm{A}}{ }^{-1 / 3}+\left(\mathrm{N}_{\mathrm{A}} / \mathrm{N}_{\mathrm{B}}\right)(\mathrm{D} / \mathrm{R})^{2}\right]^{1 / 3}}
\end{aligned}
$$

$\mathrm{n}$ is then given by eq 2.13 and

$$
\begin{aligned}
& D / R= \\
& 0.867\left[\frac{1}{2}+\frac{N_{B} / N_{A}}{\left(1+N_{B} / N_{A}\right)^{3}}-\chi_{B S}\right]^{1 / 5} N_{A}^{-8 / 11} N_{B}^{6 / 7}
\end{aligned}
$$

Here $\chi_{\mathrm{BS}}$ is a Flory-Huggins parameter describing interactions between B-monomers and solvent molecules, while $\sigma_{\mathrm{AS}}$ is the interfacial tension between pure A polymers and surrounding pure solvent (under the assumptions of strong incompatibility of $A$ and solvent we have also $\sigma_{\mathrm{AS}} \mathrm{a}^{2} / \mathrm{k}_{\mathrm{B}} \mathrm{T}=\sqrt{\chi_{\mathrm{AS}} / 6}$, for large enough $\mathrm{N}_{\mathrm{A}}$ ). From eq 2.24 for $\mathrm{N} \rightarrow \infty$ at fixed f one predicts a weak divergence of the ratio $D / R$

$$
\begin{array}{r}
D / R \propto N^{10 / 77}, \quad R / a \propto N^{134 / 231}, \begin{array}{r}
D / a \propto N^{164 / 231} \\
\bar{n} \propto N^{57 / 77}
\end{array}
\end{array}
$$

On the other hand, for good solvent conditions for the $B$ monomers and large $N_{B} / N_{A}$ (i.e.; small f) the micelles should resemble star polymers, which exhibit a scaling 65 $\mathrm{D} / \mathrm{a} \propto \mathrm{N}^{\vee}$ with $\mathrm{v} \approx 3 / 5$, which is smaller than the exponent quoted in eq 2.25.

It also is of interest how $\mu_{n}^{\circ} / \mathrm{n}-\mu_{1}^{\circ}$ itself and the critical micelle concentration scale with $\mathrm{N}$. In the limit where $\mathrm{R} / \mathrm{a} \approx\left[3 \mathrm{~N}_{\mathrm{A}}^{2}\left(\sigma_{\mathrm{A}} \mathrm{a}^{2} / \mathrm{k}_{\mathrm{B}} \mathrm{T}\right)\right]^{1 / 3}$ both terms $4 \pi \mathrm{R}^{2} \sigma / \mathrm{k}_{\mathrm{B}} T$ and $g_{\text {def }} / K_{B} T$ are of the same order, proportional to $\left.\left(\sigma_{\mathrm{AS}} \mathrm{a}^{2} / \mathrm{k}_{\mathrm{B}} \mathrm{T}\right)\right]^{5 / 3} \mathrm{~N}_{\mathrm{A}} 4 / 3$, while the term $\mathrm{g}_{\text {mix }}$ (eq 2.17) or $\mu_{\text {dil,A }}^{\circ}$ and $\mu_{\text {dil,B }}^{\circ}$ (eq 2.22) are proportional to $\mathrm{S}^{3}$ or at least $R^{3}$, i.e., proportional to $3 \mathrm{~N}_{\mathrm{A}}{ }^{2}\left(\sigma_{\mathrm{AS}} \mathrm{a}^{2}\right)$. As a consequence, we get (noting that $\mu_{1}^{\circ} \propto \mathrm{N}$ )

$$
-\operatorname{In} \phi_{\mathrm{cmc}} \propto \mathrm{N}-\text { const } \times \mathrm{N}^{1 / 3}
$$

From the work of Nagarajan and Ganesh ${ }^{27}$ one finds In $\phi_{\mathrm{cmc}} \propto \mathrm{N}^{0.7}$ (using the results of their Table 3). It may be that this smaller exponent again is an effective exponent.

Next we discuss the size distribution of the micelles for $\phi_{1}$ near $\phi_{\mathrm{cmc}}{ }^{27}$ Since $\bar{n}$ was defined from the condition that the derivative of $\Delta \mu=\mu_{\mathrm{n}}^{\circ}-\mathrm{n} \mu_{1}^{\circ}$ vanishes for $\mathrm{n}=\overline{\mathrm{n}}$, one can expand

$$
\Delta \mu(\mathrm{n})=\Delta \mu(\overline{\mathrm{n}})+\frac{1}{2} \Delta \mu^{\prime \prime}(\mathrm{n}-\overline{\mathrm{n}})^{2}+\ldots(2.27)
$$

and the size distribution function $\phi_{\mathrm{n}}$ in eq 2.9 becomes a Gaussian

$$
\phi_{\mathrm{n}}=\phi_{1}{ }^{\mathrm{n}}(\mathrm{n}-1)\left[-\Delta \mu(\overline{\mathrm{n}}) / \mathrm{k}_{\mathrm{B}} \mathrm{T}\right]\left[-\frac{1}{2 \mathrm{k}_{\mathrm{B}} \mathrm{T}} \Delta \mu^{\prime \prime}(\mathrm{n}-\overline{\mathrm{n}})^{2}\right]
$$

Since the second derivative $\Delta \mu^{\prime \prime}$ is of the same order as $\Delta \mu(\mathrm{n}) / \bar{n}^{2}$, we conclude that $\Delta \mu^{\prime \prime} \propto \sigma_{\mathrm{As}} \mathrm{a}^{2}$ if $\bar{n} \propto \mathrm{N}_{\mathrm{A}}$ or $\Delta \mu^{\prime \prime}$ $\propto \mathrm{N}^{20 / 77}$ if $\overline{\mathrm{n}} \propto \mathrm{N}^{57 / 77}$. In both cases eq 2.28 implies that the size distribution is extremely narrow, with a half- width $\Delta \mathrm{n}$ of order unity. Thus, the "pseudophase" approximation made in eq 2.10 is self-consistent.

Combining finally eqs 2.12 and 2.28 we find for $n$ near $\bar{n}$

$$
\phi_{\mathrm{n}} \propto\left(\phi_{1} / \phi_{\mathrm{cmc}}\right)^{\mathrm{n}} \exp \left[-\frac{1}{2 \mathrm{k}_{\mathrm{B}} \mathrm{T}} \Delta \mu^{\prime \prime}(\mathrm{n}-\overline{\mathrm{n}})^{2}\right]
$$

Since $\left(\phi_{1} / \phi_{c m c}\right)^{\bar{n}}=\exp \left[\bar{n} \ln \left(\phi_{1} / \phi_{c m c}\right)\right]$, the rounding of the transition $\Delta \phi_{1}$ can be estimated as

$$
\ln \left(\Delta \phi_{1} / \phi_{\text {cmc }}\right) \approx 1 / \bar{n} \text {, i.e. } \Delta \phi_{1} \approx \phi_{\text {cmc }} \text { for } \bar{n} \rightarrow \infty
$$

Since $\phi_{\mathrm{cmc}}$ tends to zero exponentially as $\mathrm{N} \rightarrow \infty, \Delta \phi_{1}$ does not shrink to zero more strongly than $\phi_{\mathrm{cmc}}$ itself, despite the large size of the aggregates that are formed.

We now return to the analogy between spherical micelles with $\mathrm{N}_{\mathrm{B}} \gg \mathrm{N}_{\mathrm{A}}$ and star polymers. ${ }^{33,74}$ This anal ogy is particularly obvious for the extreme case $\mathrm{N}_{\mathrm{A}}$ $=1$. Bug et al. ${ }^{24}$ find that in this limit eq 2.29 again holds al though $\bar{n}$ does not increase with $\mathrm{N}$ but rather is a decreasing function of $\mathrm{N}$ (or $\mathrm{N}_{\mathrm{B}}$ since $\mathrm{N}=\mathrm{N}_{\mathrm{B}}+1$ )

$$
\overline{\mathrm{n}} \propto\left(\sigma_{\mathrm{AS}} \mathrm{a}^{2} / \mathrm{k}_{\mathrm{B}} \mathrm{T}\right)^{15 / 11} \mathrm{~N}^{-3 / 11}
$$

while the width of the Gaussian, eq 2.28, scales as $N^{-2 / 11}$. These results can be used only as long as $\bar{n} \gg 1$; i.e., for not too large $\mathrm{N}$. These results are based on a liquid drop model for the micellar core. Somewhat different assumptions ${ }^{24}$ on the core region even yield the result that a cmc occurs only if $\mathrm{N}$ does not exceed a critical value $\mathrm{N}_{\mathrm{c}}$ for aggregation. This destabilization of micelles results from the effectively repulsive interactions between the B-monomers of the different chains in the corona, which are clearly unfavorable to aggregation in the good solvent region for the B-monomers. F or $\mathrm{N}_{\mathrm{A}}=1$ (or $\mathrm{N}_{\mathrm{A}}$ fixed at a finite value of order unity while $\mathrm{N}_{\mathrm{B}}$ and hence $\mathrm{N} \rightarrow \infty$ ) the unfavorable interactions cannot be balanced by the gain due to the attractions between the A-monomers in the core region.

Comparison between eqs 2.14, 2.15, 2.20, 2.22 and 2.25 , which predict a divergence of $\bar{n}$ with increasing $N$ at fixed $f=N_{A} /\left(N_{A}+N_{B}\right)$ and eq 2.31, where $N_{A}$ is fixed show again that the chain length dependence of micellar properties depends in a subtle way on how one takes the limits $\mathrm{N} \rightarrow \infty, \mathrm{N}_{\mathrm{B}} \rightarrow \infty$.

Finally, Hal perin et al. ${ }^{23,25,26}$ again exploit the analogy with star polymers ${ }^{73,74}$ but for the case of $N_{A} \ll N_{B}$ at finite $f$. They obtain a power law

$$
\bar{n} \propto N_{A}^{4 / 5}\left\{\left(\sigma_{A S} a^{2} / k_{B} T\right) / \log \left(N_{B}^{3 / 5} / \bar{n}^{2 / 15} N_{A}{ }^{1 / 3}\right)\right\}^{3}
$$

Unlike in eqs 2.22, 2.25, and $2.30 \bar{n}$ decreases with a logarithm of $N_{B}$. The $\mathrm{cmc}$ is

$$
\phi_{\mathrm{cmc}} \propto \exp \left\{-4 \pi\left(\sigma_{\mathrm{AS}} \mathrm{a}^{2} / \mathrm{k}_{\mathrm{B}} T\right) \mathrm{N}_{\mathrm{A}}^{2 / 3}\right\}
$$

I gnoring logarithmic corrections, the linear dimensions become

$$
\begin{aligned}
\mathrm{R} \propto \mathrm{N}_{\mathrm{A}}{ }^{3 / 5} \mathrm{a}\left(\sigma_{\mathrm{AS}} \mathrm{a}^{2} / \mathrm{k}_{\mathrm{B}} \mathrm{T}\right)^{2 / 5}, \\
\mathrm{D} \propto \mathrm{N}_{\mathrm{B}}{ }^{3 / 5} \mathrm{~N}_{\mathrm{A}}{ }^{4 / 25} \mathrm{a}\left(\sigma_{\mathrm{AS}} \mathrm{a}^{2} / \mathrm{k}_{\mathrm{B}} T\right)^{6 / 25}
\end{aligned}
$$

The size distribution is again given by eq 2.29 and the 

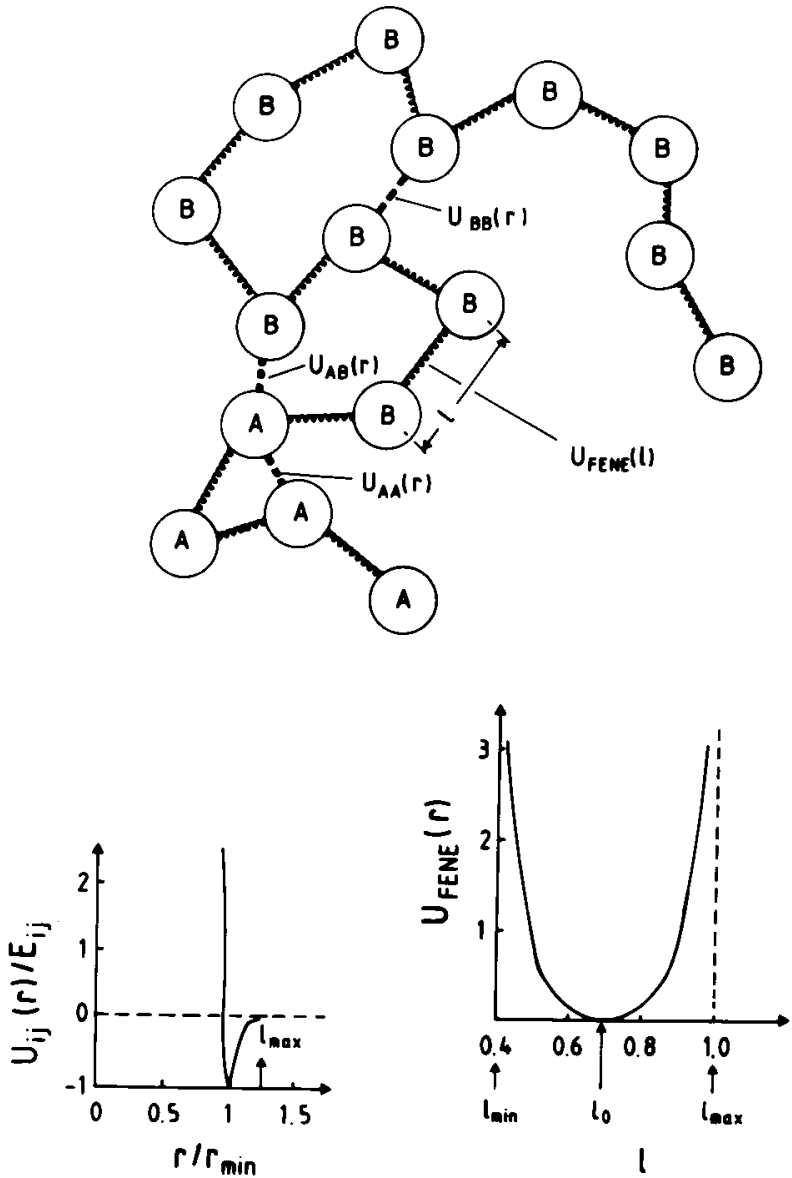

Figure 2. Schematic description of the coarse-grained bead spring model of flexible block copolymers, choosing $N_{A}=f N$ effective monomers of type $A$ and $N_{B}=(1-f) N$ effective monomers of type $B$. Nearest neighbor monomers interact with the anharmonic spring potential $U_{F E N E}(I)$ [f. text], while between any nonbonded pair of monomers we assume shortrange Morse potentials $U_{i j}(r)$, where indices $i$ and $j$ stand for $A$ or $B$, respectively. Note that these Morse potentials are nonzero only if $r<r_{\text {cutoff }}=1$.

micelles are very monodisperse since

$$
\delta \mathrm{n} / \overline{\mathrm{n}} \propto \overline{\mathrm{n}}^{-3 / 4} \propto \mathrm{N}_{\mathrm{A}}^{-3 / 5}
$$

Halperin et al.23,25,26 obtain a power law decay for the density profile of B-monomers in the corona

$$
\phi_{B}(r) \propto \bar{n}^{2 / 3}(a / r)^{4 / 3}, \quad R \leq r \leq R+D
$$

Equation 2.36 is analoguous to the density profile of star polymers with $\bar{n}$ arms. ${ }^{73}$ Since one must have $4 \pi \int_{R}^{R+D}$ $\phi_{B}(r) r^{2} d r=a^{3} \bar{n} N_{B}$ one finds for $D \gg R$ readily that $D^{5 / 3}$ $\propto \bar{n}^{1 / 3} N_{B}$, which gives eq 2.34 when one uses eq 2.32. The main difference between star polymers and micelles with $N_{B} \gg N_{A}$ is that for star polymers $\bar{n}$ is given by the chemical synthesis while for micelles $\bar{n}$ is selfadjusting by minimization of the free energy of a micelle.

\section{Computer Simulations: Model and Simulations Technique}

F or the sake of computational efficiency, we simulate a model of a block copolymer solution, where only the polymer chains are taken into account explicitly; the selective solvent is treated only implicitly via suitable effective potentials $U_{A A}(r), U_{A B}(r)$, and $U_{B B}(r)$ between the different types (A, B) of effective monomers (Figure 2). The advantage of this si mplification is that relatively large systems (containing up to 1024 polymer molecules) can be simulated with reasonable computational effort. In view of this simplification, it does not make much sense to aim for a description of chemical details of the block copolymers, of course, rather the latter are treated on a coarse-grained level as well, where one imagines that several subsequent chemical monomers along the real polymer chain are integrated into one effective segment connecting effective monomers (Figure 2 ). In fact, this coarse-graining along the chemical sequence of the polymer chain is a standard tool of polymer simulation. ${ }^{46}$ We describe an effective bond by an anharmonic spring connecting the two neighboring effective monomers, using the FENE (finitely extensible nonlinear elastic) potential $65,67,75-83$ for the length I of the effective bond

$$
\begin{array}{r}
U_{\text {FENE }}(I)=-\frac{1}{2} K\left(I_{\max }-I_{0}\right)^{2} \operatorname{In}\left[1-\left(I-I_{0}\right)^{2} /\right. \\
\left.\left(I_{\max }-I_{0}\right)^{2}\right]
\end{array}
$$

This potential is approximately harmonic near its minimum at $I=I_{0}, U_{\text {FENE }}(I) \approx 1 / 2 \mathrm{~K}\left(I-I_{0}\right)^{2}$, but diverges both for $I=I_{\max }$ and for $I=I_{\min }=2 I_{0}-I_{\max }$, cf. Figure 2 (lower part). Following our previous work, $65,67,75-83$ we choose $I_{\max }=1$ as our unit of length and $I_{0}=0.7$, so that $I_{\min }=0.4$. To ensure that most of the bonds have a length close to $\mathrm{I}_{0}$ the effective spring constant is taken as $\mathrm{K}=40 \mathrm{~K}_{\mathrm{B}} \mathrm{T}$. N ote that we choose the parameters $\mathrm{K}$, $I_{\max }$ and $I_{0}$ the same for all effective bonds, irrespective of whether we deal with a bond connecting two effective monomers of type $A$, or type $B$, or the junction bond connecting $\mathrm{A}$ and $\mathrm{B}$.

Between any nonbonded pair of effective monomers we use Morse potential $s^{65}$

$$
\begin{aligned}
& U_{\mathrm{ij}}(r)= \mathrm{E}_{\mathrm{ij}}\left\{\exp \left[-2 \alpha\left(r-r_{\text {min }}\right)\right]-\right. \\
&\left.\quad \exp \left[-\alpha\left(r-r_{\min }\right)\right]\right\}+C, \quad r<r_{\text {cutoff }} \\
& U_{\mathrm{ij}}(r)=0, \quad r>r_{\text {cutoff, }}, \quad\{i j\}=\{A, B\}
\end{aligned}
$$

The strength $E_{i j}$ of these potentials is chosen such that the solvent is a good solvent for the B-monomers and a bad solvent for the A-monomers, at the chosen temperature $T=1$ (choosing al so the normalization $\mathrm{k}_{\mathrm{B}}=1$ )

$$
2.2 \leq \mathrm{E}_{\mathrm{AA}} \leq 6.5, \quad \mathrm{E}_{\mathrm{AB}}=\mathrm{E}_{\mathrm{BB}}=1.0
$$

Also some runs have been made for the alternative choice $\mathrm{E}_{\mathrm{BB}}=0.1, \mathrm{E}_{\mathrm{AB}}=0.1$, leading to a larger effective Flory-Huggins parameter $\chi \propto \mathrm{E}_{\mathrm{AA}}+\mathrm{E}_{\mathrm{BB}}-2 \mathrm{E}_{\mathrm{AB}}$ between $\mathrm{A}$ and $\mathrm{B}$ monomers.

The $\Theta$-temperature for the corresponding homopolymer model with $\mathrm{E}=1$ is $^{77} \Theta \approx 0.62$, i.e., $\mathrm{E} / \Theta \approx 1.61$ : Thus, for eq 3.4 we indeed have $\mathrm{E}_{\mathrm{BB}} / \mathrm{T}=1<\mathrm{E} / \Theta \approx 1.6$ $<\mathrm{E}_{\mathrm{AA}} / \mathrm{T}=\mathrm{E}_{\mathrm{AA}}$. The constant $\mathrm{C}$ in eq 3.2 is chosen such that the potential $U_{i j}(r)$ is regular at $r=r_{\text {cutoff. }}$ The position $r=r_{\min }$ where the potential $U_{\mathrm{ij}}(r)$ has its minimum is chosen also to be of order unity, similar but not identical to $I_{0}$, namely $r_{\min }=0.8$. The parameter $\alpha$ controls the range of the potential.

We have found it convenient for the use of very fast link-cell algorithms ${ }^{76}$ to have an extremely short-range potential, and thus we choose $\alpha=24$. A sketch of this potential is also given in Figure 2 (lower left part). One 
then can choose $r_{\text {cutoff }}=1$; i.e., $I_{\max }=1$ can al so be used as cell size of the link-cell algorithm. ${ }^{76} \mathrm{~N}$ ote that we choose also the parameters $\alpha, r_{\min }$, and $r_{\text {cutoff }}$ to be the same for all these types of pair interactions, (AA), (AB), and $(B B)$, respectively. The only difference allowed between these interactions is their strength (eq 3.4).

Typically, we choose as a simulation volume a cubic box of linear dimension $L=32$ with periodic boundary conditions, containing in total 4096 monomers; given our choice of units this means a density of $\phi=1 / 8$ (note that typical melt densities in these units correspond to $0^{69} \phi$ $\approx 1.5$ to $\phi \approx 2.0$ ). Unlike the analytical theories of section 2, where one typically considers the transition at the $\mathrm{cmc}$ varying $\phi$ at fixed energy parameters, we find it more convenient in the simulation to study the transition at the $\mathrm{cmc}$ keeping $\phi$ fixed (at the value quoted above) and varying $\mathrm{E}_{\mathrm{AA}}$ : in this way the task of comparing different choices of $\mathrm{N}$ is much easier rather than keeping $\mathrm{E}_{\mathrm{AA}}$ fixed and varying $\phi$.

In fact, from eqs 2.26 and 2.33 one recognizes that, at fixed $E_{A A}, \phi_{\mathrm{C} C \mathrm{c}}$ decreases dramatically with increasing $\mathrm{N}$. As is obvious from eq 2.33, the variation of $\phi_{\mathrm{cmc}}$ with $\mathrm{N}$ can be compensated by an appropriate variation of $\sigma_{\mathrm{AS}} / \mathrm{K}_{\mathrm{B}} \mathrm{T}$ (and hence $\mathrm{E}_{\mathrm{AA}}$, which controls this interfacial free energy). If we would study cases where $\phi_{\mathrm{cmc}}$ is extremely small, we would need to study huge simulation boxes, since finite size effects are only avoided if the total number of micelles at $\phi=\phi_{\mathrm{cmc}}$ in the simulation volume is much larger than unity. A simulation of huge boxes is impractical, however, since equilibration requires that the chains can diffuse through the whole simulation box, which takes a time $\tau_{\mathrm{D}} \approx \mathrm{L}^{2} /\left(6 \mathrm{D}_{\mathrm{N}}\right)$, where $D_{N}$ is the self-diffusion constant of the chains. Since our Monte Carlo simulation algorithm ${ }^{75-77}$ corresponds to a Rouse-like motion of the chains, ${ }^{84,85}$ we have $\left.D_{N} \propto\right|_{0} ^{2} /$ $\mathrm{N}$, and thus we see that with $\mathrm{L}=32, \mathrm{~N}=32$ al ready diffusion times on the order of $10^{5}$ Monte Carlo steps (MCS) per effective monomer result. When large micelles have formed, the equilibration of the micellar size distribution may require even much larger times, since it is required that polymers "evaporate" from one micelle and then diffuse to some other micelle at great distance in the simulation box, and this process needs to be repeated many times. However, in order that a polymer chain can get out of a micellar aggregate an activation free energy barrier needs to be crossed. Already in the work on short block copolymers $(\mathrm{N}=4)$ with $\mathrm{f}=1 / 2$, it was found ${ }^{65}$ that, for concentrations $\phi$ exceeding $\phi_{\mathrm{cmc}}$ distinctly, the occurrence of metastable large micelles was a severe problem, hampering the establishment of full equilibrium. Although in the present work, observation times up to $10^{7}$ MCS per effective monomer were used, we must expect similar equilibration problems.

3.1. Simulation Results and their Analysis. To make contact with experiments and other simulations, where $\phi_{\mathrm{cmc}}$ is probed by varying the density $\phi$ of the monomers in the system at fixed $E_{A A}$, we present such data for our model in Figure 3. Here one simply probes the density (or concentration, respectively, when one associates the free volume in the simulation box with the solvent molecules) $\phi_{1}$ of monomers that belong to copolymers but do not belong to aggregates (we shall call such nonaggregated copolymers "unimers" in the following). As a criterion whether a chain bel ongs to an aggregate or not, we simply monitor its interchain $A-A$ interactions in the configuration that is analyzed: if there are no such interchain A-A interactions present

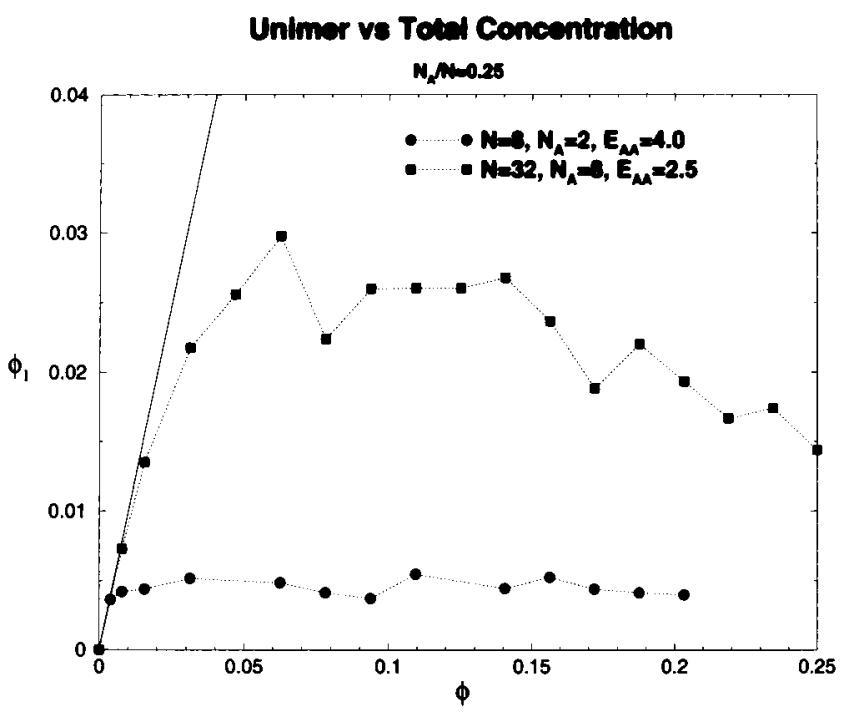

Figure 3. Variation of the concentration $\phi_{1}$ of monomers in the copolymers (not in aggregates) plotted vs total monomer concentration for $\mathrm{N}=8$ (circles) and $\mathrm{N}=32$ (squares), respectively, choosing $\mathrm{E}_{\mathrm{AA}}=4.0$ for $\mathrm{N}=8$ and $\mathrm{E}_{\mathrm{AA}}=2.5$ for $\mathrm{N}=32$. The straight line represents the ideal solution behavior of the copolymer solution, $\phi_{1}=\phi$.

(i.e., all other A-monomers not bel onging to the considered chain are further than a distance $r_{\text {cutoff }}$ away, cf. eqs 3.2 and 3.3), the chain is counted as an unimer.

The behavior seen for $\mathrm{N}=8$ is rather typical: for very small concentrations $\phi$, we have no aggregates, $\phi=\phi_{1}$, while for $\phi>\phi_{\text {cmc. }} \phi_{1}$ stays al most constant. In this case $\phi_{\mathrm{cmc}} \approx 0.004$ which means that in our box of volume $32^{3}$ we have only about 16 chains present near $\phi_{\mathrm{cmc}}$, and hence finite size effects clearly are expected. Now $\phi_{\mathrm{cmc}}$ can be increased by decreasing the strength of the attractive energy $E_{A A}$ between the A-monomers. I $n$ fact, choosing $\mathrm{E}_{\mathrm{AA}}=2.5$ instead of $\mathrm{E}_{\mathrm{AA}}=4.0$ and choosing four times longer chains, we observe that $\phi_{\mathrm{cmc}}$ can be raised to about $\phi_{\mathrm{cmc}} \approx 0.03$. Although the theoretical expectation is that $\phi_{\mathrm{cmc}}$ decreases rapidly with increasing chain length $\mathrm{N}$, this decrease here is offset by the decrease of $\mathrm{E}_{\mathrm{AA}}$ : this observation al ready indicates that it is very convenient to choose $\mathrm{E}_{\mathrm{AA}}$ rather than $\phi$ as a control parameter. On the other hand, it is somewhat disturbing that the deviation from the straight line $\phi_{1}$ $=\phi$ is more gradual for $\mathrm{N}=32$ than for $\mathrm{N}=8$. This deviation means that small clusters of copolymers (dimers, trimers, ...) occur at concentrations $\phi_{1}<\phi_{\text {cmc }}$ in appreciable concentrations already, before large micelles are formed at $\phi=\phi_{\text {cmc. }}$. From eq 2.9 it is clear that for $\phi_{1}<\phi_{\mathrm{cmc}}$ we expect a size distribution of aggregates that is monotonically exponentially decreasing with aggregate size $n$, and this was in fact already observed in our earlier work ${ }^{65}$ and similar other studies. ${ }^{63,64}$ The unimer concentration for $N=32$ is found to decrease with $\phi$ for $\phi_{1}>\phi_{\mathrm{cmc}}$ again. Such a decrease was also found in earlier studies ${ }^{63-65}$ and is attributed to copolymer-micelle interaction effects. Such interaction effects might influence somewhat the prefactors of the power laws for the various properties of the micelles reviewed in section 2 but should not affect universal exponents or other qualitative features of the behavior. Thus, we consider these interaction effects tolerable. Since a pronounced decrease of $\phi_{1}$ with $\phi$ for $N=32$ only starts for $\phi \approx 0.14$, and the number of chains in the simulation box for $\phi=\phi_{\mathrm{cmc}} \approx 0.03$ is still rather small (about 30), we henceforth fix $\phi=0.125$ (which 
a)

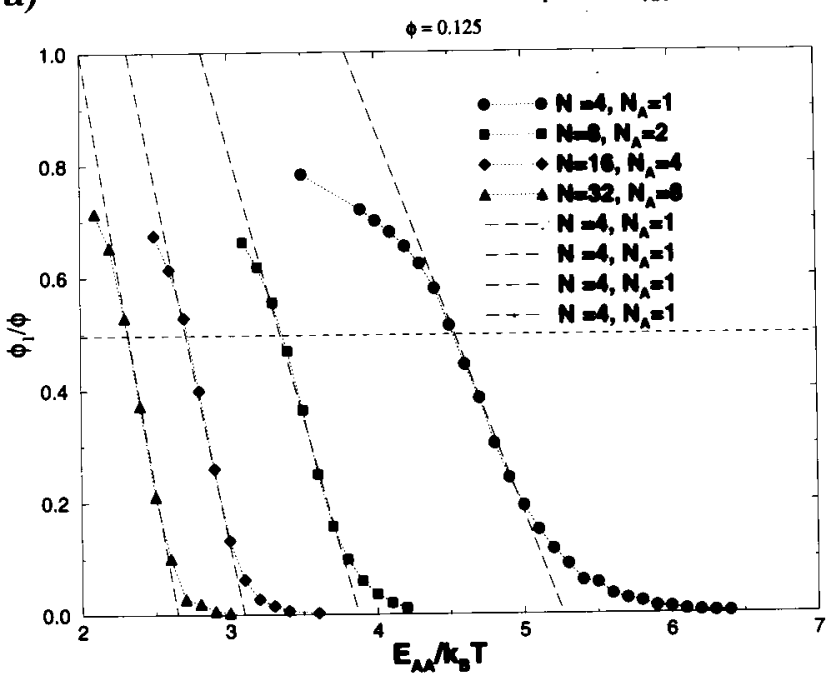

b)

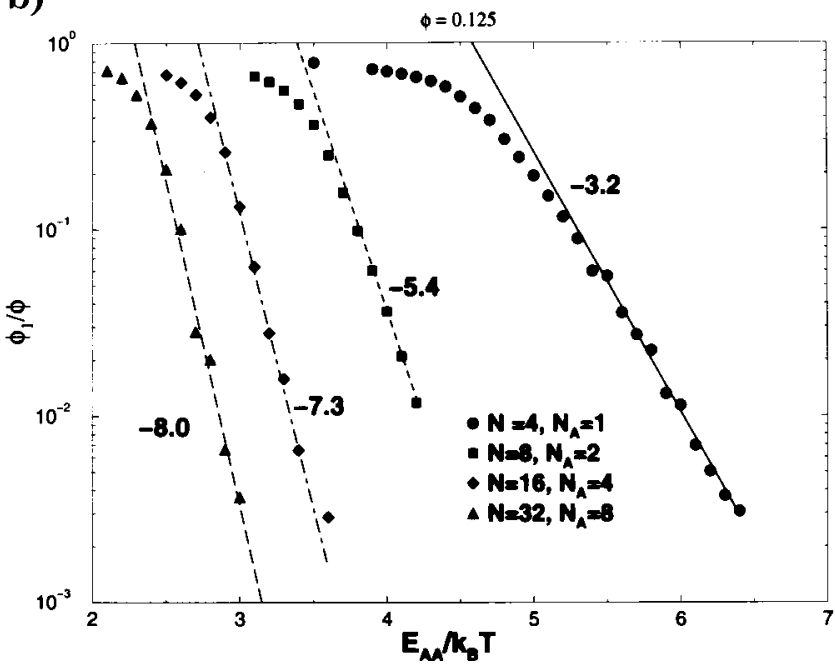

Figure 4. Unimer fraction $\phi_{1} / \phi$ plotted vs $E_{A A} / k_{B} T$, for $f=1 / 4$ and four choices of $\mathrm{N}$ as indicated in the figure. Part $\mathrm{a}$ is a linear-linear plot, showing the estimation of $E_{A A}^{c}$ via the condition $\phi_{1} / \phi=1 / 2$, while part $\mathrm{b}$ is a semilog plot, showing that $\phi_{1}$ decays exponentially with $\mathrm{E}_{\mathrm{AA}} / \mathrm{k}_{\mathrm{B}} \mathrm{T}, \phi_{1} \propto \exp \left[-\mathrm{C}_{\mathrm{N}} \mathrm{E}_{\mathrm{AA}} /\right.$ $\left.k_{B} T\right]$. The numbers quoted at the straight lines of part $b$ denote the constants $C_{N}$. In part $a$, the estimation of the width of the transition region $\Delta \mathrm{E}_{\mathrm{AA}} / \mathrm{K}_{\mathrm{B}} \mathrm{T}$ is also indicated.

means, we still have 128 chains in our simulation box for our largest chain length $\mathrm{N}=32$, while for the smaller values of $\mathrm{N}$ chosen here the numbers of chains are cor respondingly larger), and study micelle formation varying $\mathrm{E}_{\mathrm{AA}}$ at fixed $\phi=0.125$ in the remainder of the paper.

Figure 4 gives a corresponding plot of the unimer concentration $\phi_{1}$ vs $E_{A A} / k_{B} T$, for the four chain lengths $\mathrm{N}=4,8,16$ and 32 studied in the present paper. In the thermodynamic limit, $\mathrm{N} \rightarrow \infty$, we would expect from the theories of section 2, which describe micelle formation as a sharp phase transition, that $\phi_{1} / \phi$ as a function of $\mathrm{E}_{\mathrm{AA}} / \mathrm{k}_{\mathrm{B}} \mathrm{T}$ develops toward a step function, i.e., $\phi_{1} / \phi=r_{1}$ for $E_{A A} / k_{B} T<E_{A A}^{C} / k_{B} T$ and $\phi_{1} / \phi=r_{2}$ for $E_{A A} / k_{B} T>$ $E_{A A}^{c} / k_{B} T$, with the constant $r_{1}$ close to unity, and the constant $r_{2}$ close to zero. However, Figure 4 shows that the transition is rather gradual for all values of $\mathrm{N}$ that were studied. Thus, we empirically define the critical value $E_{A A}^{c}$ of micelle formation from the condition $\phi_{1} / \phi$
$=1 / 2$, and from the slope of the straight line of the $\phi_{1} / \phi$ vs $E_{A A} / k_{B} T$ curve for $E_{A A}=E_{A A}^{C}$ we define a width $\Delta E_{A A}$ as shown in Figure 4. From this analysis we find, remembering $\mathrm{k}_{\mathrm{B}} \mathrm{T}=1$, for $\mathrm{N}=4: \mathrm{E}_{\mathrm{AA}}^{\mathrm{C}}=4.5, \Delta \mathrm{E}_{\mathrm{AA}} \approx$ 1.6; for $\mathrm{N}=8, \mathrm{E}_{\mathrm{AA}}^{\mathrm{C}}=3.38, \Delta \mathrm{E}_{\mathrm{AA}} \approx 1.0$; for $\mathrm{N}=16$ : $\mathrm{E}_{\mathrm{AA}}^{\mathrm{C}}$ $=2.75, \Delta \mathrm{E}=0.85$; and finally, for $\mathrm{N}=32$ : $\mathrm{E}_{\mathrm{AA}}^{\mathrm{C}}=2.23$, $\Delta \mathrm{E}_{\mathrm{AA}} \approx 0.7$. It is seen that the transition does become slightly sharper with increasing $N$, but since $E_{A A}^{C}$ also decreases distinctly with increasing $\mathrm{N}$, any extrapolation toward $\mathrm{N} \rightarrow \infty$ clearly would be very subtle. Ultimately we expect that $E_{A A}^{c}$ at fixed $\phi$ develops toward $E_{A A}^{\Theta}$ for $N \rightarrow \infty$, the value of the energy at the $\Theta$-point for the A-blocks, which is ${ }^{77} \mathrm{E}_{\mathrm{AA}}^{\Theta} \approx 1.61$.

An alternatve estimation of $E_{A A}^{C}$ is possible from the observation (Figure $4 b$ ) that $\phi_{1} \propto \exp \left[-\mathrm{C}_{N} E_{A A} / k_{B} T\right]$, where the constant $\mathrm{C}_{\mathrm{N}}$ al so distinctly increases with $\mathrm{N}$. Extrapolating to the energy where this exponential law would be equal to $\phi$ gives an alternative estimate for $E_{A A}^{c}$, which is $E_{A A}^{c}=4.6,3.4,2.7$ and 2.3, for $N=4,8$, 16 and 32 , respectively.

Within the errors of these procedures both ways of estimating $\mathrm{E}_{\mathrm{AA}}^{\mathrm{c}}$ are reasonably well consistent with one another.

To be able to compare our results with the theories for section 2, we also have obtained the size distribution of the micelles for various choices of $\mathrm{E}_{\mathrm{AA}}$ (Figure 5) as well as the resulting mean aggregate weight $\bar{n}$ defined as

$$
\overline{\mathrm{n}}=\sum_{\mathrm{n}=2}^{\infty} \mathrm{n}^{2} \phi_{\mathrm{n}} / \sum_{\mathrm{n}=2}^{\infty} \mathrm{n} \phi_{\mathrm{n}}
$$

Defining the mole fractions $\varphi_{n}$ of aggregates in terms of their volume fractions $\phi_{\mathrm{n}}$ as $\varphi_{\mathrm{n}}=\phi_{\mathrm{n}} / \mathrm{n}$, we obtain the mean aggregation number $\langle\mathrm{n}\rangle$ of the micelles and the fluctuation of their size $\sigma$ as usual

$$
\langle\mathrm{n}\rangle=\sum_{\mathrm{n}=2}^{\infty} \mathrm{n} \varphi_{\mathrm{n}} / \sum_{\mathrm{n}=2}^{\infty} \varphi_{\mathrm{n}}, \sigma^{2}=\sum_{\mathrm{n}=2}^{\infty} \mathrm{n}^{2} \varphi_{\mathrm{n}} / \sum_{\mathrm{n}=2}^{\infty} \varphi_{\mathrm{n}}-\langle\mathrm{n}\rangle^{2}
$$

From Figure 5 we see that for all choices of $\mathrm{N}$ the size distribution is clearly monotonically decreasing for $\mathrm{E}_{\mathrm{AA}}$ distinctly smaller than $E_{A A}^{c}$, develops a flat shoulder for $E_{A A}$ near $E_{A A}^{c}$, and becomes clearly bimodal for $E_{A A}$ distinctly larger than $E_{A A}^{C}$.

Thus, we find that the Gaussian description for the aggregate size distribution (eq 2.29) is a good description in the concentrated region off the critical micelle concentration $\phi_{\mathrm{cmc}}$, but it is not applicable close to $\phi_{\mathrm{cmc}}$, at least not for the chain lengths $\mathrm{N}$ that were accessible here. But even more disturbing is the fact, that neither the aggregate size $\bar{n}$ nor the mean aggregation number $\langle\mathrm{n}\rangle$ show a clear tendency to increase with N. Thus, for our short chains, no indication of the asymptotic laws are seen, that should hold according to all theoretical predictions of section 2 (Figures 6 and 7). It is clear that all these averages $\bar{n},\langle n\rangle$ are still affected by the tail of the exponentially decreasing part of the distribution $\left\{\phi_{2}\right.$, $\left.\phi_{3}, \ldots\right\}$. Given the observation that the shoulder in the aggregate size distribution $\mathrm{P}(\mathrm{n})$ develops at sizes $\mathrm{n} \geq$ 10 , it seems to us that it would make more sense of not counting small aggregates (with $n=2,3, \ldots$ ) among the micelles, but rather treat the block copolymer solution (that coexists with the micelles at $\phi=\phi_{\text {cmc }}$ ) not as an ideal solution, and all ow for clusters of copolymers with 

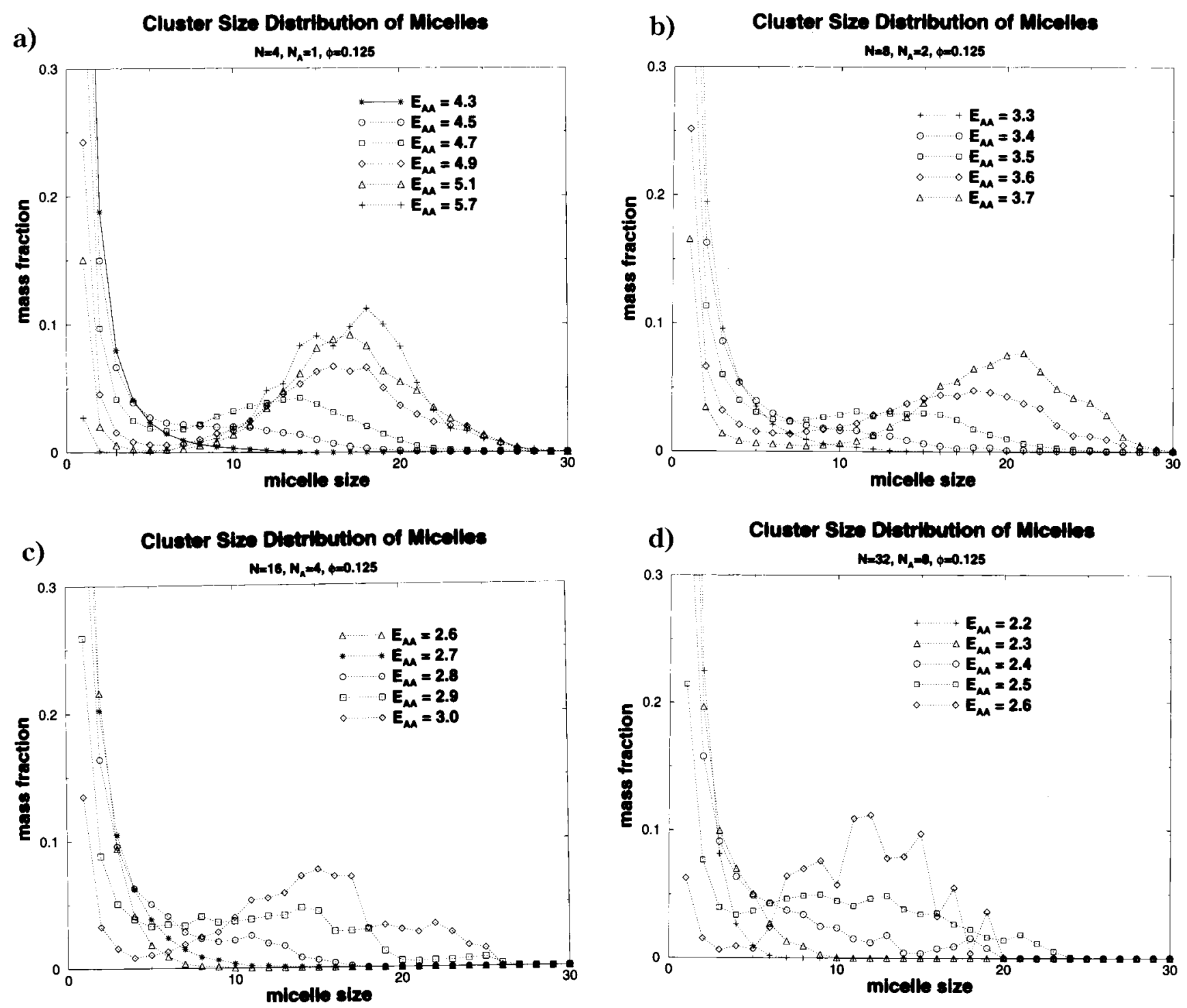

Figure 5. Cluster size distribution of the micelles for $N=4(a), N=8(b), N=16$ (c), and $N=32$ (d).

$\mathrm{n}=2,3, \ldots$ in that solution already. This means that only aggregates exceeding a cutoff size $n_{\text {cutoff }}$ are counted as micelles, and hence eq 3.6 needs to be replaced by

$$
\langle\mathrm{n}\rangle=\sum_{\mathrm{n}>\mathrm{n}_{\text {cutoff }}} \mathrm{n} \varphi_{\mathrm{n}} / \sum_{\mathrm{n}>\mathrm{n}_{\text {cutoff }}} \varphi_{\mathrm{n}}
$$

This modified definition is included in Figure 6b: Now one recognizes that near $E_{A A}^{C}$ there is a somewhat more rapid increase of $\langle n\rangle$ from $n_{\text {cutoff }}+1$ to the size $n_{\max }$ where $\phi_{n}$ is maximal. However, for values of $E_{A A} / k_{B} T$ that are only slightly larger than $E_{A A}^{c}$ there seems al ready a problem with equilibration, as the fluctuations in $\langle n\rangle$ and ultimately the decrease of $\langle n\rangle$ with increasing $E_{A A} / k_{B} T$ shows. And the problem remains that near $\mathrm{E}_{A A}^{\mathrm{C}}$ the micelle size $\langle\mathrm{n}\rangle$ does not significantly increase with increasing chain length $\mathrm{N}$, in contrast with the theoretical predictions.

Also the behavior of the fluctuation $\sigma$ is interesting (Figure 7): it is seen that $\sigma$ is still rather small at $\mathrm{E}_{A A}^{c}$, again almost independent of $\mathrm{N}$, and monotonically increasing with $E_{A A} / k_{B} T$, until a maximum is reached at values of $E_{A A}$ somewhat larger than $E_{A A}^{c}$. Since the aggregation transition at $\phi_{\mathrm{cmc}}$ is treated by the theory of section 2 as a (rounded) first-order phase transition, one would expect that fluctuation quantities such as $\sigma$ pick up a contribution from a (rounded) $\delta$ function singularity describing fluctuations between the two coexisting phases (copolymer solution vs micellar solution, respectively). In fact, such peaks describing rounded first-order phase transitions have been seen in simulations of many corresponding models, e.g., Potts models. ${ }^{86,87}$ One might argue that the lack of any such peak in Figure 7 at $\mathrm{E}_{\mathrm{AA}}^{\mathrm{c}}$ is simply due to the fact that also $\sigma$ is dominated by contribution from small aggregates ( $\mathrm{n}$ $=2,3, \ldots)$ for $E_{A A}$ near $E_{A A}^{c}$ : therefore these aggregates are not strongly fluctuating in their number, and thus $\sigma$ is relatively small and is not much affected by the twophase fluctuations. However, this argument is not applicable, since for the data shown in Figure 7 only contributions for $\mathrm{n}>\mathrm{n}_{\text {cutoff }}$ were included.

We now turn to the geometric characteristics of the micelles that are formed. Figure 8 shows radial density distribution functions of micelles, separately for the core and corona regions of the micelles, so that the radii $\mathrm{R}$ and $\mathrm{S}$ (cf. Figure 1) can be inferred. From the high values of the density in the core, we can see that for $\mathrm{N}$ $=4$ and $\mathrm{N}=8$ indeed melt densities are reached, as was assumed in most of the theories of section 2. For $\mathrm{N}$ $=16$ and $\mathrm{N}=32$, however, the density in the core 
a)

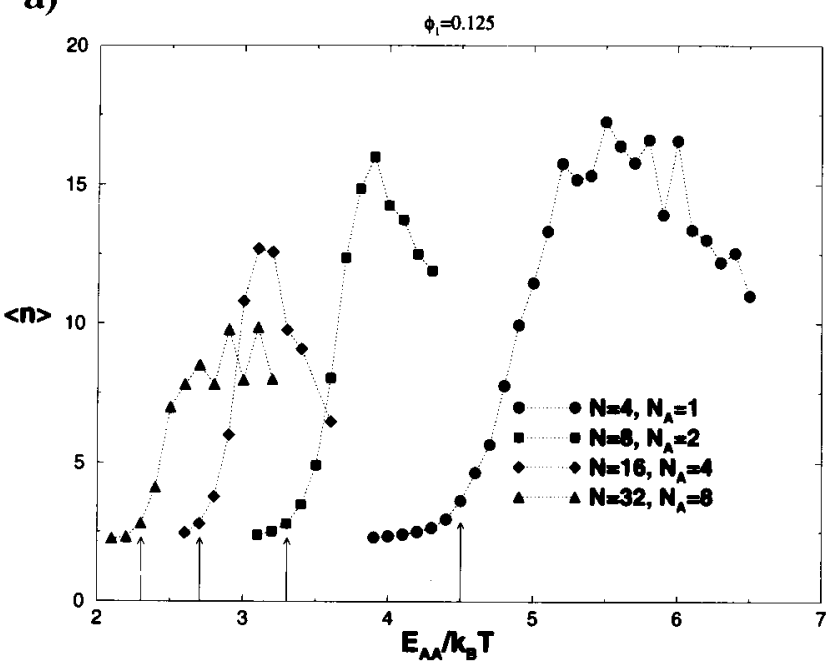

b)

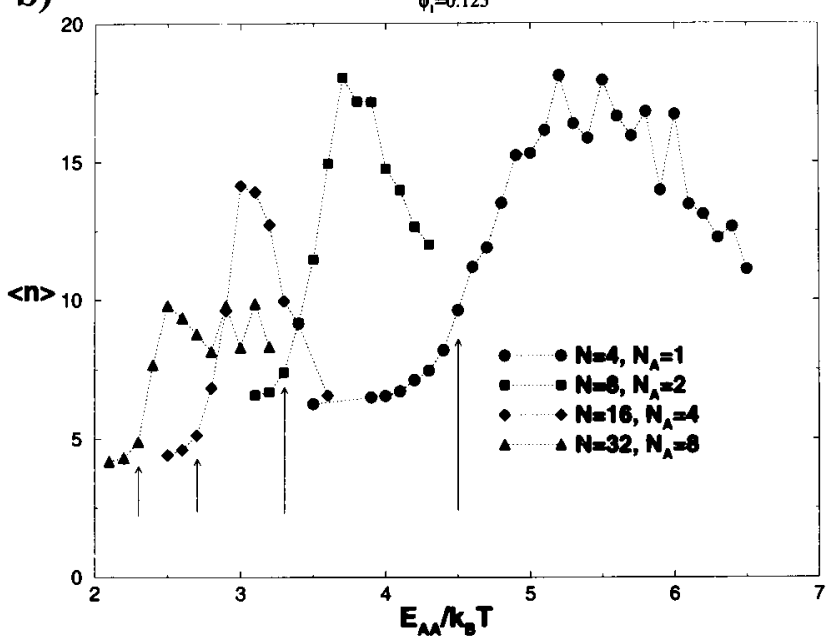

Figure 6. (a) Mean aggregation number $\langle\mathrm{n}\rangle$ (eq 3.5) plotted vs $E_{A A} / k_{B} T$ for $N=4,8,16$, and 32. Arrows show estimates for $E_{A A}^{C}$ as obtained in Figure 4. (b) Same as part a but for the modified definition, eq 3.7, with $\mathrm{n}_{\text {cutoff }}$ chosen in the minimum of $\phi_{\mathrm{n}}$ (Figure 5).

corresponds to a concentrated polymer solution only, and not to a melt. This behavior is reasonable, however, since the values of $E_{A A}^{C}$ for $N=16$ and $N=32$ show that $E_{A A}^{c}$ no longer is very far away from $E_{A A}^{\Theta}$, and hence, there develops a nonzero concentration of the "solvent" (or free volume, respectively) in the core as well.

Again it is noticeable that our results do not reproduce the theoretical prediction of a strong increase of the core radius with $\mathrm{N}$. Rather we can see that there is only a very weak increase of the core radius with $\mathrm{N}$. This is a corollary of our result that the aggregation number $\langle n\rangle$ does not increase with $\mathrm{N}$ : Therefore, there is no chain stretching possible; instead, the chains in the core resemble collapsed configurations, and we expect only an increase $\mathrm{R} \propto \mathrm{N}^{1 / 3}$ of the core radius, very different from the predictions $R \propto N^{2 / 3}$ (eq 2.14) of de Gennes, ${ }^{14}$ $\mathrm{R} \propto \mathrm{N}^{0.58}$ of Nagarajan and Ganesh ${ }^{27}$ (eq 2.25), or $\mathrm{R} \propto$ $\mathrm{N}^{3 / 5}$ (eq 2.34) of Halperin et al. ${ }^{23,25,26}$ Equation 2.13, however, is compatible with our result, since a relation $\mathrm{R} \propto \mathrm{N}^{1 / 3}$ indeed implies that $\langle\mathrm{n}\rangle$ does not increase with $\mathrm{N}_{\mathrm{A}}$, as we have found here.

When we turn to the corona region, we see that for $\mathrm{N}$ $=4$ and $\mathrm{N}=8$ rather high densities are reached despite
Standard Deviation of the Maan Aggregation Number

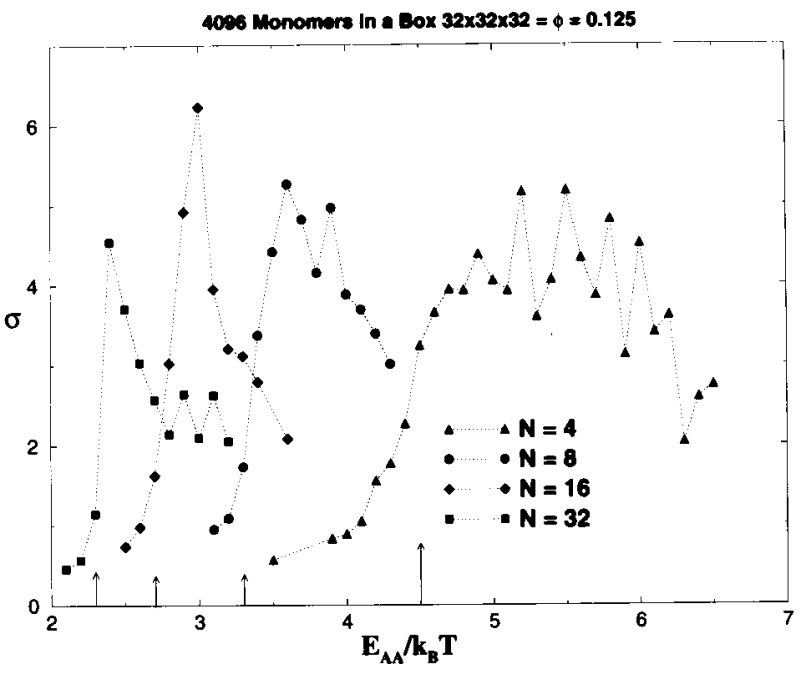

Figure 7. Standard deviation $\sigma$ of $\langle n\rangle$ (eq 3.6) plotted vs $\mathrm{E}_{\mathrm{AA}} /$ $k_{B} T$ for $\mathrm{N}=4,8,16$, and 32. Arrows show estimates for $E_{A A}$ as obtained in Figure 4.

the repulsive interactions. The high density in the cores, together with chain connectivity, requires relatively many $B$-monomers to be present in the $A-B$ interfacial region. Since we have chosen $E_{A B}=E_{B B}(=1)$, the repulsive interaction between $A-B$ pairs is not stronger than between $B-B$ pairs, and hence, it is a matter of concern whether sharp $\mathrm{A}-\mathrm{B}$ interfaces can be expected. Of course, since the combination $E_{A B}-\left(E_{A A}^{C}+E_{B B}\right) / 2$ $=1 / 2-E_{A A}^{C} / 2<0$, we do expect a strong tendency of phase separation in dense systems, since in the regime where the Morse potential function is negative (Figure 2) a net repulsive $A-B$ interaction always results. However, only if the product $N\left(E_{A A}^{C}-1\right) \phi \gg 1$ do we expect extremely sharp interfaces between the A-rich core and the B-rich corona, and this regime is hardly reached here. Thus, the radius of the core and the width of the A-B interfacial region are of comparable size. In contrast, the theories of section 2 almost always have assumed that the interfacial width is negligible in comparison with both the core radius and the linear dimension of the corona. This is again an indication that one must be careful when one wishes to compare simulations to scaling theories, which hold only in particular asymptotic limits and not over the full range of chain lengths. To investigate this problem further, in Figure 8, we have also included data for the choice $E_{A B}=0.1, E_{B B}=0.1$, for which the effective $\chi$ parameter between $A$ and $B$ monomers is larger (see section 3 ), and hence, the interface between the core and the corona should be sharper. However, this enhancement of incompatibility has little effect: the B-chains in the corona are slightly more stretched, to avoid infavorable contact with A monomers.

One must also consider, of course, that the minimum value that the interfacial width can reach for a very incompatible system is of the order of the length of an effective monomer, which is 0.7 for our choice of units. Therefore, interfacial widths much narrower than seen in Figure 8 cannot really be expected.

In Figure 9 the density profiles for the different chain lengths are compared to each other, choosing now the value of $E_{A A} / k_{B} T$ in each case such that it coincides with $E_{A A}^{C} / k_{B} T$ within error. One sees that there is only a rather small increase of both the size of the core and 
a)

Core and Corona for $\mathrm{N}=8, \phi=0.125$

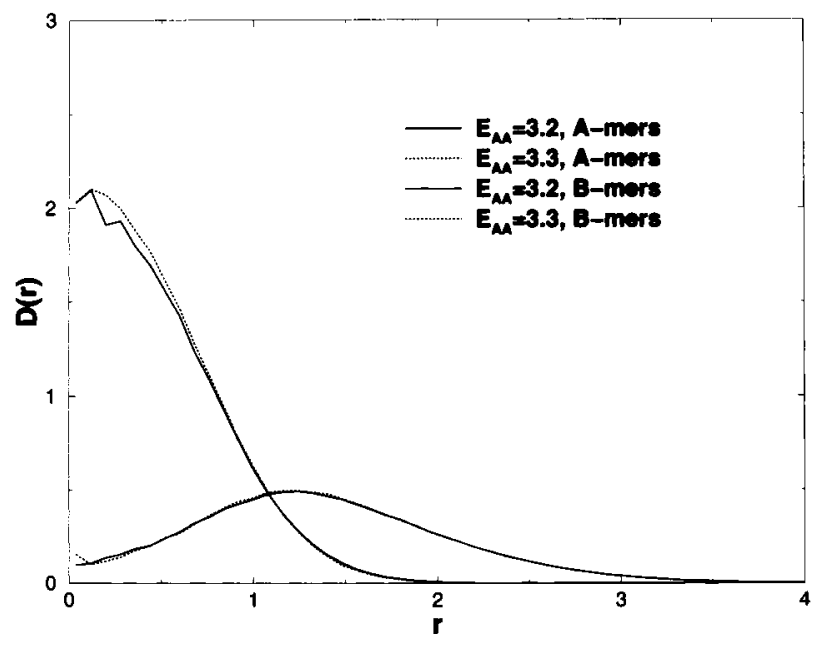

b)

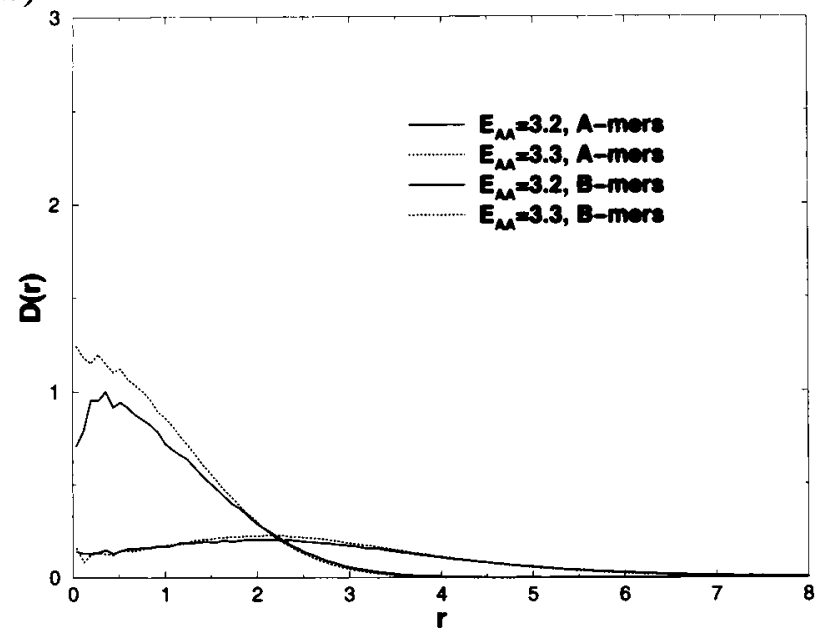

c)

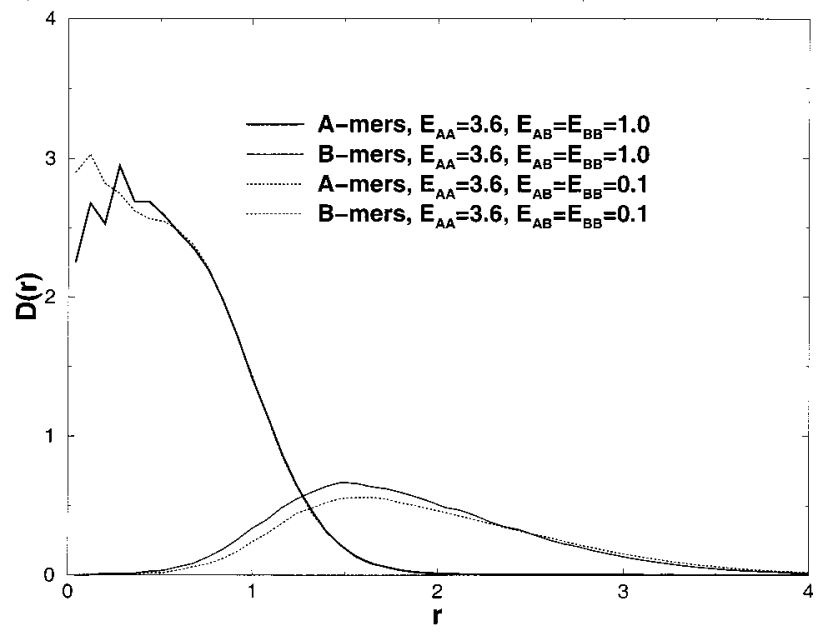

Figure 8. (a) Density distribution function $D(r)$ of micelles for $\mathrm{N}=8$ and two choices of $\mathrm{E}_{\mathrm{AA}} / \mathrm{k}_{\mathrm{B}} \mathrm{T}$ as indicated in the figure. Contributions from the core and from the corona are shown separately. (b) Same as part a but for $\mathrm{N}=32$. Only micelles with aggregation numbers $\mathrm{n}>\mathrm{n}_{\text {cutoff }}$ have been included in the analysis. (c) Same as part a, but for the choice $E_{A A}=3.6$, and two choices of $E_{A B}$ and $E_{B B}$; namely $E_{A B}=E_{B B}=1.0$ and $E_{A B}=E_{B B}=0.1$, to show that there is only a weak dependence on the parameter $E_{A B}-\left(E_{A A}+E_{B B}\right) / 2$, which in this case is -1.3 or -1.75 , respectively.
Density Radial Distribution in Micelles

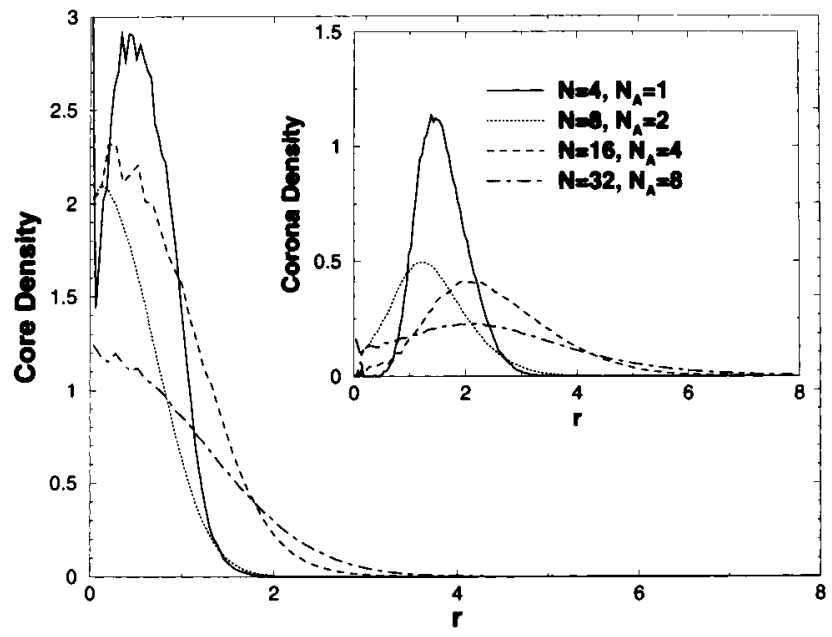

Figure 9. Radial density distribution function of A-mers (main part) and B-mers (insert), comparing data at different chain lengths as indicated. The energy $E_{A A} / k_{B} T$ was chosen as $4.5(\mathrm{~N}=4), 3.3(\mathrm{~N}=8), 2.7(\mathrm{~N}=16)$, and $2.3(\mathrm{~N}=32)$, respectively, corresponding approximately to $E_{A A}^{c} / k_{B} T$.

the size of the corona with increasing N, while the decay in the tails of the distribution for large $r$ becomes definitely slower with increasing $\mathrm{N}$.

Finally, Figure 10 presents results for the radial distribution function $\mathrm{G}(\mathrm{r})$ of the centers of masses of the micelles. Since there is an excluded volume interaction between the micelles, $G(r)$ is essentially zero up to the micelle radius and then slowly grows up to unity; this value is reached at about twice the micelle radius, since for larger distances the interactions among micelles become negligible. Therefore, in thermal equilibrium $G(r)$ is expected to be constant and equal to unity for these distances, and this expectation is corroborated by the data for $\mathrm{N}=8$ and energies $\mathrm{E}_{\mathrm{AA}} / \mathrm{K}_{\mathrm{B}} \mathrm{T} \leq 3.8$ and for $\mathrm{N}=32$ and energies $\mathrm{E}_{\mathrm{AA}} / \mathrm{k}_{\mathrm{B}} \mathrm{T} \leq 2.5$. For larger values of $E_{A A} / k_{B} T$, however, $G(r)$ exhibits structure on much larger length scales, which we interpret as a clear evidence for the lack of proper equilibration; that is, since the micelles formed in the system are essentially frozen, the density fluctuations in the system are also frozen in over the time scale of oberservation time, and hence the condition $G(r \rightarrow \infty) \rightarrow 1$ is violated. This result reiterates our conclusion that the region of energies where $\langle n\rangle$ in Figure $6 \mathrm{~b}$ decreases with increasing $\mathrm{E}_{\mathrm{AA}} /$ $k_{B} T$ needs to be discarded, since equilibrium could not be established properly.

\section{Concluding Remarks and Comparison with Experiment}

In the present investigation micelle formation of block copolymers in selective solvents was studied by Monte Carlo simulation of a coarse-grained model, varying at fixed monomer density in the system and fixed composition $f=N_{A} /\left(N_{A}+N_{B}\right)$ the chain length $N$. Micelle formation then can be studied conveniently choosing $E_{A A} / k_{B} T$ as control variable, keeping all other energy parameters $\left(E_{A B} / k_{B} T, E_{B B} / k_{B} T\right.$, the spring constant $\mathrm{K} / \mathrm{K}_{\mathrm{B}} \mathrm{T}$ ) as well as geometrical parameters characterizing the model $\left(I_{0}, I_{\min }, r_{\min }, r_{\text {cutoff }}\right)$ at fixed values. The purpose of our study was to el ucidate how geometrical properties of the micelles as well as their mean aggregation number $\langle\mathrm{n}\rangle$ depends on the chain length $\mathrm{N}$, 
a) Radial Distribution Function of Micelles

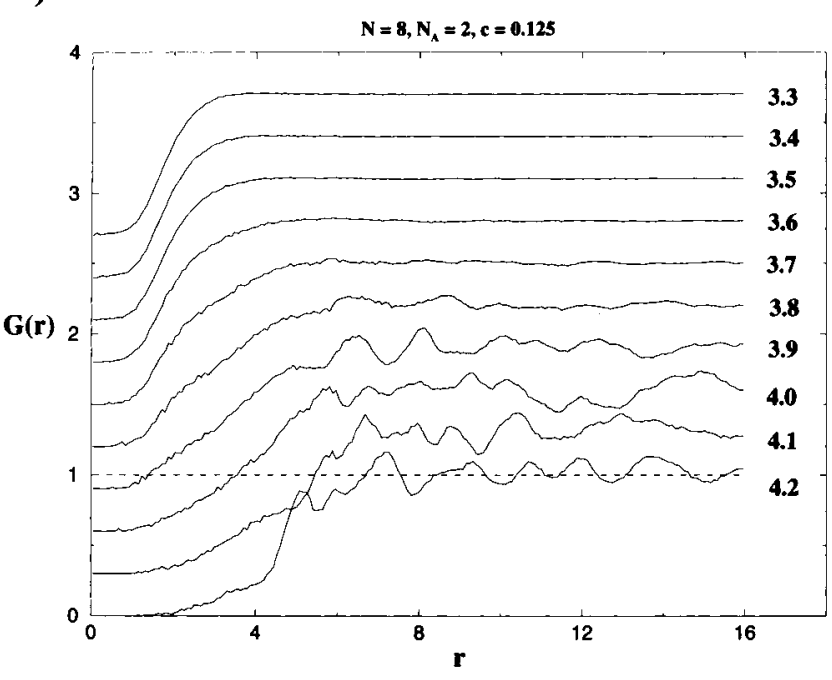

b) Radial Distribution Function of Micelles

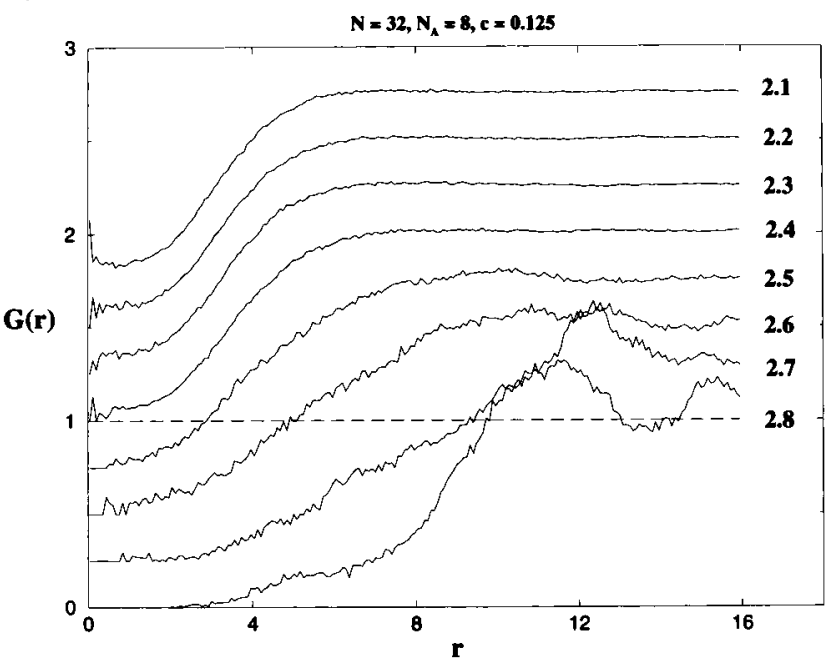

Figure 10. Radial distribution function $G(r)$ of the centers of mass of the micelles plotted vs $r$, for $N=8(a)$ and $N=32(b)$. Various choices of $E_{A A} / k_{B} T$ are included, as indicated. Note that only the largest value of $E_{A A} / k_{B} T$ has the ordinate scale as shown, while the origin for the other choices is displaced in units of 0.3 (a) and 0.25 (b), to avoid that the curves are too much messed up.

in the regi me of chain lengths which is accessible to the simulations. Various theoretical predictions on micelle formation of block copolymers are available only for the limit $\mathrm{N} \rightarrow \infty$ and mostly for the case of sharp interfaces between core and corona. These theories have in common that they predict that the mean aggregation number $\langle\mathrm{n}\rangle$ of block copolymers forming a micelle increase with some power law of $\mathrm{N}$ for large $\mathrm{N}$; the exponent of this power law differs in the various theories. It is interesting to check whether these power laws-or different ones-can also be seen for our rather small values of $\mathrm{N}$.

Of course, due to the slow relaxation of macromolecular aggregates computer simulations can achieve thermal equilibration only for rather short chain lengths, and while we are able to vary $\mathrm{N}$ over a reasonable range-almost a decade, $\mathrm{N}=4$ to $\mathrm{N}=32$-it is clear that even our largest chain length corresponds still to much smaller molecular weights than are used in experimental studies (comparing to experiment one must note that each effective bond in our model corre sponds to a group of 3-5 successive chemical monomers of a real polymer). Therefore, a comparison of our results with experimental data can only be of a qualitative character. We summarize some of the most pertinent experimental findings in the following.

Cogan et al..$^{38}$ have studied polystyrene (PS)-poly(ethylene oxide) (PEO) diblock copolymers in cyclopentane by small-angleX-ray and neutron scattering, using two systems, characterized by different degrees of polymerization, namely PEO/PS $=65 / 80$ and $170 / 1730$, respectively. The aim of this study was to test the starpolymer-like micelle model. ${ }^{23}$ While they find that the model is useful for the case where the PS blocks in the corona are very long, the model does not work for the $65 / 80$ bl ock copolymers.

Noting that one should take five polystyrene repeat units as a statistical unit, Cogan et al. ${ }^{38}$ attribute the failure of the starlike model to the fact that 16 statistical units per chain in the corona are not enough to apply this scaling model, and these short chains in the corona are found to be more stretched than expected from the theory. A be at al. ${ }^{39}$ consider micelle formation from even smaller molecules, namely nonionic surfactants of the type $C_{m} P E_{n}$ \{poly(oxyethylene) ethers with alkyl chain lengths $m=12,14$, and 16 , at $n=20$, while the poly(oxyethylene) chain length was al so varied from $n=10$ to $n=40$ for $m=16\}$. These authors studied both the aggregation number and the micellar size at the $\mathrm{cmc}$ and found that for small $m$ and/or small $n$ the aggregation number stays almost constant (at a value of about 40) and also the micellar diameter for small $m$ stays almost constant. These findings have some qual itative similarity with the results of the present paper.

Zhang et al. ${ }^{40}$ studied scaling relations and coronal dimensions for polystyrene-b-poly(acrylic acid) block copolymers in aqueous solution, using degrees of polymerization for the PS block (which forms the core) from 170 to 1400, and for the poly(acrylic acid) from 33 to 310 , respectively. Since this polymer is a polyelectrolyte, chain conformations in the corona are highly extended and cannot be compared to our model, which is applicable to neutral polymers only. However, nevertheless, it is interesting that an empirical scaling relation $\mathrm{R} \propto \mathrm{N}_{\mathrm{A}}{ }^{0.5} \mathrm{~N}_{\mathrm{B}}{ }^{-0.15}$ for the core radius is found, i.e., a dependence on the length $\mathrm{N}_{\mathrm{A}}$ of the insoluble block that is much weaker than predicted by the theories that we have mentioned in section 2 .

A particularly thorough study ${ }^{41}$ of poly(styrene-b-4vinylpyridine) (PS-P4VP) copolymer, on the other hand, reached the conclusion that a scaling relation for the aggregation number $\mathrm{n} \propto \mathrm{N}_{\mathrm{A}}{ }^{2} \mathrm{~N}_{\mathrm{B}}-0.5$ holds, while the corona dimension probed by dynamic light scattering exhibited a law $D \propto n^{1 / 5} \mathrm{~N}_{B}{ }^{3 / 5}$. H owever, these author ${ }^{41}$ made their measurements at concentrations far above the $\mathrm{cmc}$, due to problems of locating the cmc ("anomalous micellization" occurs at very low polymer concentrations). Förster et al. ${ }^{41}$ observe their power law already for systems containing relatively short Ablocks: their smallest degree of polymerization is $N_{P S}$ $=25$, corresponding to about five statistical segments only, and for this system with $\mathrm{N}_{\mathrm{P} 4 \mathrm{VP}}=293$, an aggregation number as small as $\mathrm{n} \approx 6$ is found. In fact, reanalyzing also some small surfactant data from the literature Förster et al. ${ }^{41}$ suggest that their scaling behavior can be followed to extremely short $\mathrm{N}_{\mathrm{A}}$, such as $N_{A}=3$. However, data by Schuch et al. ${ }^{44}$ for 
polyisobutylene-block-poly(methacrylic acid) in water confirm this scaling behavior for large degree of polymerization, while for small $\mathrm{N}_{\mathrm{A}}$ some systematic deviations from the above power law seem to occur. This study ${ }^{44}$ (as well as ref 42) has the merit that data close to the cmc could be taken, and it was shown that the micelle formation near the $\mathrm{cmc}$ is spread out over about a full decade in concentration. Finally, we mention the work of Willner et al. ${ }^{45}$ who studied the structure of micelles varying the length of the block in the corona over a wide range and showed that the starlike model is applicable only for $N_{B} \gg N_{A}$, while the theory of Nagarajan and Ganesh ${ }^{27}$ works better for not so large $\mathrm{N}_{\mathrm{B}}$.

Thus, the conclusion of all these studies, taken together, is that there are several regimes where somewhat different power laws apply if $\mathrm{N}_{\mathrm{A}}, \mathrm{N}_{\mathrm{B}}$ are both large, but when $\mathrm{N}_{\mathrm{A}}, \mathrm{N}_{\mathrm{B}}$ are small, the behavior does not really seem universal. However, all studies agree that for small $N_{A}, N_{B}$ also the aggregation numbers in the micelles are fairly small, and the transition at the $\mathrm{cmc}$ is rather gradual. These two latter observations are in agreement with the conclusions of our simulations. However, our results do not agree with the suggestion of Förster et al. ${ }^{41}$ that for strongly segregated blocks the core radius scales as $\mathrm{R} \propto \mathrm{N}_{\mathrm{A}}$ \{ which is an inevitable consequence of $\mathrm{n} \propto \mathrm{N}_{\mathrm{A}}{ }^{2} \mathrm{~N}_{\mathrm{B}}-0.5$ together with $\mathrm{nN}_{\mathrm{A}}=4 \pi$ $(R / a)^{3} / 3$, eq 2.13 \} down to very small values of $N_{A}$. Instead, our findings imply that for very small $\mathrm{N}_{\mathrm{A}}$ there is another regime, where $R \propto N_{A}{ }^{1 / 3}$ and hence $n$ does not increase with $\mathrm{N}_{\mathrm{A}}$ yet. There do exist other data which may be compatible with our results, however. ${ }^{39}$

Unfortunately, we are unable to present any quantitative comparison with the theoretical predictions, simply because there is qualitative disagreement between our simulations and all the theories available, which address a regime (very long chains, core linear dimensions that are much larger than the interfacial width), that cannot be reached in the simulations. This is al ready seen from Figure 5-when the size distribution function of the aggregates starts to become bimodal, the peak corresponding to the formation of micelles appears in the range $10 \leq n \leq 20$, and there is no tendency either in the peak position (Figure 5) or in the average $\langle n\rangle$ (Figure 6) to increase with chain length $N$. Unexpectedly, there is rather a weak trend in the opposite direction, in particular if the very small aggregate sizes $(n=2,3, \ldots$, up to the minimum of the bimodal distribution) are excluded from the average $\langle n\rangle$ (compare Figure 6b with Figure 6a). While it is clear that for a quantitative precision of the various theories reviewed in section 2 one needs to consider very large $\mathrm{N}$, it is not at all evident from the derivation of these theories that they break down completely for intermediate and small values of $\mathrm{N}$. To make this point as clear as possible, we have briefly reviewed the main physical assumptions and approximations made in the various theories. In our understanding, these theories do not offer an explanation yet for a completely different regime of behavior for the range of short chain lengths as investigated here, where the core radius scales as $R$ $\propto N_{A} 1 / 3$, and hence $\langle n\rangle$ is independent of $N_{A}$.

This conclusion is corroborated by our results for the other properties of the micelles as well: since only a few chains aggregate together in a micelle, the A-rich cores form a collapsed configuration of the A-parts of the chains they contain, rather than forming stretched configurations, and so the increase of the core radius $\mathrm{R}$
(Figures 1,8 , and 9) with $\mathrm{N}$ is extremely weak. At the same time, the thickness of the interface between the A-rich core and the B-rich corona is of about the same order as the core radius - which also is no surprise given the fact that this interface forms with a rather small number $\langle\mathrm{n}\rangle$ of chains only.

One explanation of these surprising findings could be that the simulation is trapped in metastable states this in fact does happen for fairly large values of $E_{A A} /$ $\mathrm{k}_{B} T$ distinctly larger than the critical value $E_{A A}^{c} / k_{B} T$ for micelle formation: then small and medium-size aggregates are formed rather fast in the initial stages of the simulation and then stay, since there are not enough "unimers" left in the system in order to provide further equilibration. Only if chains can escape from aggregates again and diffuse freely as unimer from one aggregate to another one, can the micellar size distribution equilibrate well. However, this lack of equilibration for large $E_{A A} / k_{B} T$ is clearly seen from many criteria$\langle n\rangle$ is no longer monotonically increasing with $E_{A A} / k_{B} T$ but develops a maximum and therefore huge fluctuations occur; also the fluctuation $\sigma$ (Figure 7) develops a rather erratic behavior, and the radial distribution function $G(r)$ of the center of masses of the micelles develops random structure at large distances. All these characteristic behaviors seen for large $E_{A A} / k_{B} T$ find a consistent interpretation in terms of frozen-in micellesbut at the same time none of these characteristics is present near $E_{A A}^{c} / k_{B} T$, and we feel that the data for $\langle n\rangle$ are well equilibrated up to the value of $E_{A A} / k_{B} T$ where the first peak of $\langle n\rangle$ occurs-which also includes the region where the first peak of $\sigma$ occurs. Of course, for small $\mathrm{N}$ the transition from unimers (+ dimers, trimers etc.) to micelles is not a sharp transition, but it is smeared. Therefore, different criteria for locating $E_{A A}^{c} / k_{B} T$, necessarily must give slightly different results; we have found that a criterion based on the decrease of the relative unimer concentration $\phi_{1} / \phi$ (Figure 4) is most efficient to use. However, if we would use other criteria-such as taking the position of maximal slope in the $\langle n\rangle$ vs $\mathrm{E}_{\mathrm{AA}} / \mathrm{k}_{\mathrm{B}} \mathrm{T}$ curve or $\sigma$ vs $\mathrm{E}_{\mathrm{AA}} / \mathrm{k}_{\mathrm{B}} T$ curve or even the position of the first peak of $\sigma$-the conclusions about the existence of a new regime of micellar behavior where $\mathrm{R} \propto \mathrm{N}_{\mathrm{A}}{ }^{1 / 3}$ would not be al tered.

While we have no convincing theoretical explanation for the existence of this regime to offer, we hope that our study will lead to theoretical developments comple menting the existing theories such that they are also able to explain our results. After all, such systems of relatively short block copolymers in selective solvents should also be easily accessible in the laboratory.

Acknowledgment. A.M. acknowledges partial support from the Deutsche F orschungsgemeinschaft (DFG) under Grant No. 436 BUL 113/92, and A.B. under Grant No. SF B262/D2. A.B. also acknowledges the hospitality from the University of Mainz during a stay where this work was initiated and thanks the NSF for financial support through Grant No. CRG-612418. Furthermore, A.B. is grateful to Prof. S. D. Mahanti for useful discussions.

\section{References and Notes}

(1) Israelachvili, J . I ntermol ecular and SurfaceF orces; Academic Press: London, 1991; Chapter 17.

(2) Tanford, C. The Hydrophobic Effect: Formation of Micelles and Biological Membranes; Wiley: New York, 1980.

(3) Lindman, B.; Wennerström, H. Top. Curr. Chem. 1980, 87, 
(4) Mittal, K. L.; Lindman, B., Eds. Surfactants in Solution; Plenum: New York, 1984

(5) Degiorgio, V., Corti, M., Eds. Physics of Amphiphiles: Micelles, Vesicles, and Microemulsions; North-Holland: Amsterdam, 1985.

(6) Zorf, R., Ed. Surfactant Solutions: New Methods of I nvestigations; New York, 1987

(7) Evans, D. F.; Wennerström, H. The Colloi dal Domain When Physics, Chemistry, Biology and Technology Mee; VCH Publishers: Weinheim, Germany, 1994.

(8) Hall, D. G.; Pethica, B. A. In Nonionic Surfactants; Schick, M. J ., Ed.; Marcel Dekker: New York, 1967.

(9) Meier, D. J . Polym. Sci., Part, C. 1969, 26, 81

(10) Mukerjee, P. J . Phys. Chem. 1972, 76, 565.

(11) Meier, D. J . In Block and Graft Copolymers; Burke, J . J ., Ed.; Syracuse University Press: Syracuse, NY, 1973; p 105.

(12) Ruckenstein, E.; Nagarajan, R. Phys. Chem. 1975, 79, 2622.

(13) I sraelachvili, J. N.; Mitchell, D.J .; Ninham, B. W. J . Chem. Soc., Faraday Trans. 1 1976, 72, 1525

(14) de Gennes, P. G. In Solid State Physics; Liebert, J., Ed. Academic Press: New York, 1978. Suppl. 14, p 1.

(15) Nagarajan, R.; Ruckenstein, E.J . Colloid I nterface Sci. 1979, $71,580$.

(16) Stillinger, F. H.; Ben-Naim, A. J Chem. Phys. 1981, 74, 2510.

(17) Ben-Shaul, A.; Gelbart, W. M. J . Phys. Chem. 1982, 86, 316.

(18) Leibler, L.; Orland, H.; Wheeler, J . C. J . Chem. Phys. 1983, 79,3550

(19) Noolandi, J .; Hong, K. M. Macromolecules 1983, 16, 1443.

(20) Gelbart, W. M.; Ben-Shaul, A.; McMullen, W. E.; Masters, A. Phys. Chem. 1984, 88, 861.

(21) Nagarajan, R. Adv. Colloid Interface Sci. 1986, 26, 205

(22) Goldstein, R. E. J . Chem. Phys. 1986, 84, 3367.

(23) Halperin, A. Macromolecules 1987, 20, 2943.

(24) Bug, A. L. R.; Cates, M. E.; Safran, S. A.; Witten, T. A. J . Chem. Phys. 1987, 87, 1824

(25) Marques, C. M.; J oanny, J .-F.; Leibler, L. Macromolecules 1988, 21, 1051.

(26) J oanny, J.-F.; Leibler, L. In New Trends in Physics and Chemistry of Polymers; Lee, L.-H., Ed.; Plenum: New York, 1989; p 503.

(27) Nagarajan, R.; Ganesh, K. J . Chem. Phys. 1989, 90, 5843.

(28) Brown, R. A.; Masters, A. J .; Price, C.; Yuan, X. F. In Comprehensive Polymer Science; Allen, G., Ed.; Pergamon Press: Oxford, England, 1989; Vol. 2, p 155.

(29) Ligoure, C. Macromolecules 1991, 24, 2968.

(30) Nagarajan, R. Colloids Surf. A: Physicochem. Eng. Aspects 1993, 71, 39.

(31) Aggarwal, S., Ed. Block Copolymers; Plenum Press: New York, 1970.

(32) Tuzar, S.; Kratochvil, P. Adv. Colloid Interface Sci. 1976, 6, 201.

(33) Price, C. In Development of Block Copolymers I; Goodman, I., Ed.; Applied Science Publ: London, 1982; p 39.

(34) Riess, G.; Bahadur, P.; Hurtrez, G. Encyclopedia of Polymer Science and Engineering; Wiley: New York, 1985; p 324.

(35) Bates, S. F.; Fredrickson, G. H. Annu. Rev. Phys. Chem. 1990, $41,525$.

(36) Binder, K. Adv. Polym. Sci. 1994, 112, 181.

(37) Bahadur, P.; Sastry, N. V.; Marti, S.; Riess, G. Colloid Surf. 1985, 16, 337.

(38) Cogan, K.; Gast, A. P.; Capel, M. Macromolecules 1991, 24, 6512.

(39) Abe, M.; Uchiyama, H.; Yamaguchi, T.; Suzuki, T.; Ogino, K. Langmuir 1992, 8, 2147.

(40) Zhang, L.; Barlow, R. J .; Eisenberg, A. Macromolecules 1995, 28,6055

(41) Förster, S.; Zisenis, M.; Wenz, E.; Antonietti, M. J . Chem. Phys. 1996, 104, 9956.

(42) Szczubialka, K.; Tshikawa, K.; Marishima, Y. Langmuir 1999, 15, 454

(43) Wu, C.; Gao, j. Macromolecules 2000, 33, 645

(44) Schuch, H.; Klingler, J .; Rossmanith, P.; Frechen, T.; Gerst, M.; Feldthusen, J .; Müller, A. H. E. Macromol ecules 2000, 33, 1734.

(45) Willner, L.; Poppe, A.; Allgaier, J .; Monkenbusch, M.; Lindner, P.; Richter, D. Europhys. Lett. 2000, 51, 628.

(46) Binder, K., Ed. Monte Carlo and Molecular Dynamics Simulations in Polymer Science; Oxford University Press: New York, 1995.

(47) Binder, K., Ciccotti, G., Ed. Monte Carlo and Molecular Dynamics of Condensed Matter Systems; Italian Physical Society: Bologna, Italy, 1996.
(48) Landau, D. P.; Binder, K. A Guideto MonteCarlo Simulations in Statistical Physics; Cambridge University Press: Cambridge, England, 2000

(49) Haile, J. M.; O'Connell, J. P. J . Phys. Chem. 1984, 88, 6363. Woods, M. C.; Haile, J. M.; O'Connell, J. P. J Phys. Chem 1986, 90, 1875. Karaborni, S.; O'Connell, J . P.J . Phys. Chem. $1990,94,2624$.

(50) Watanabe, K.; Ferrario, M.; Klein, M. L. J . Phys. Chem. 1988, 92, 819. Watanabe, K.; Klein, M. L. J . Phys. Chem. 1989, 93 6897. Shelley, J . C.; Sprik, M.; Klein, M. L. Langmuir 1993, 9, 916.

(51) Larson, R. G. J . Chem. Phys. 1988, 89, 1642; 1989, 91, 2479; 1992, 96, 7904: Macromolecules 1994, 27, 4198.

(52) Gunn, J. R.; Dawson, K. A. J . Chem. Phys. 1989, 91, 6393.

(53) Smit, B.; Hilbers, P. A. J .; Esselink, K.; Rupert, L. A. M.; van Os, N. M.; Schlijper, A. G. Nature (London) 1990, 348, 624; J . Phys. Chem. 1991, 95, 6361. Smit, B.; Esselink, R.; Hilbers, P. A.J .; van Os, N. M.; Rupert, L. A. M.; Szleifer, I. Langmuir 1993, 9, 9. Karaborni, S.; van Os, N. M.; Esselink, K.; Hilbers, P. A. J . Langmuir 1993, 9, 1175. Karaborni, S.; Esselink, K.; Hilbers, P. A. J .; Smit, B.; Karthäuser, J .; van Os, N. M.; Zana, R. Science 1994, 266, 254.

(54) Wang, Y.; Mattice, W. L.; Napper, D. H. Langmuir 1993, 9 , 66. Adriani, P.; Wang, Y.; Mattice, W. L. J . Chem. Phys. 1994, 100,7718

(55) Stauffer, D.; J an, N.; Pandey, R. Physica A 1993, 198, 401. Stauffer, D.; J an, W.; He, Y.; Pandey, R. B.; Marangoni, D. G.; Smith-Palmer, T. J . Chem. Phys. 1994, 100, 6934.

(56) Böcker, J .; Brickmann, J .; Bopp, P.J . Phys. Chem. 1994, 98 , 712

(57) Rector, D. R.; van Swol, F.; Henderson, J . R. Mol. Phys. 1994 82, 1009.

(58) Bernardes, A. T.; Henriques, V. B.; Bisch, P. M. J . Chem. Phys. 1994, 101, 645.

(59) Wijmans, C. M.; Linse, P. Langmuir 1995, 11, 3748.

(60) Desplat, J. . .; Care, C. M. Mol. Phys. 1996, 87, 441. Care, C. M. J . Chem. Soc., Faraday Trans. 1987, 83, 2905.

(61) Mackie, A. D.: Onur, K.: Panagiotopoulos, A. Z. J . Chem Phys. 1996, 104, 3718. Mackie, A. D.; Panagiotopoulos, A. Z.; Szleifer, I. Langmuir 1997, 13, 5022.

(62) Palmer, B. L.; Liu, J . Langmuir 1996, 12, 746.

(63) von Gottberg, F. K.; Smith, K. A.; Hatton, T. A. J . Chem. Phys 1997, 106, 9850.

(64) Nelson, P. H.; Rutledge, G. C.; Hatton, T. A. J . Chem. Phys. 1997, 107, 10777

(65) Viduna, D.; Milchev, A.; Binder, K. Macromol. Theory Simul. 1998, 7, 649.

(66) Bhattacharya, A. J . Phys.: Condens. Matter, in press.

67) Milchev, A.; Binder, K. Langmuir 1999, 15, 3232.

(68) Milchev, A.; Paul, W.; Binder, K. Macromol. Theory Simul. 1994, 3, 915.

(69) Flory, P. J . Principles of Polymer Chemistry; Cornell University Press: Ithaca, NY, 1953.

(70) Helfand, E.; Tagami, Y.J . Chem. Phys. 1971, 56, 3592

(71) de Gennes, P. G. Scaling Concepts on Polymer Physics: Cornell University Press: Ithaca, NY, 1979.

(72) Noolandi, J.; Hong, K. M. Macromolecules 1982, 15, 482

(73) Daoud, M.; Cotton, J. P. J . Phys. (Paris) 1982, 43, 531.

(74) Witten, T. A.; Pincus, P. A. Macromolecules 1986, 19, 2509.

(75) Milchev, A.; Paul, W.; Binder, K. J . Chem. Phys. 1993, 99 4786 .

(76) Gerroff, I.; Milchev, A.; Binder, K.; Paul, W. J . Chem. Phys. 1993, 98, 6526.

(77) Milchev, A.; Binder, K. Macromol . Theory Simul. 1994, 3, 915.

(78) Milchev, A.; Binder, K. Macromolecules 1996, 29, 343

(79) Binder, K.; Milchev, A.; Baschnagel, J . Annu. Rev. Mater. Sci. 1996, 26, 107

(80) Pandey, R. B.; Milchev, A.; Binder, K. Macromol ecules 1997 90, 1194.

(81) Yamakov, V.; Milchev, A.; Binder, K. J . Phys. II Fr. 1997, 7 , 1123.

(82) Milchev, A.; Binder, K. Eur. Phys. J . B. 1998, 3, 477.

(83) Milchev, A.; Yamakov, V.; Binder, K. Phys. Chem. Chem. Phys. 1999, 1, 2083; Europhys. Lett. 1999.

(84) Rouse, P. E. J . Chem. Phys. 1953, 21, 1272.

(85) Doi, A.; Edwards, S. F. Theory of Polymer Dynamics; Clarendon: Oxford, England, 1987.

(86) Challa, M. S. S.; Landau, D. P.; Binder, K.Phys. Rev. B 1986, 34, 1841 .

(87) Binder, K. Rep. Prog. Phys. 1997, 60, 487.

MA000645] 